
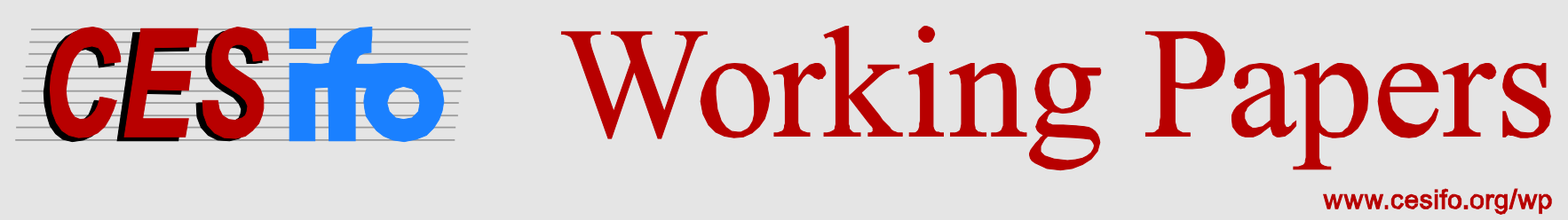

\title{
Compliance Dynamics Generated by Social Interaction Rules
}

\author{
Vilen Lipatov
}

CESIFO WORKING PAPER NO. 4767

CATEGORY 13: BEHAVIOURAL ECONOMICS

APRIL 2014
An electronic version of the paper may be downloaded
- from the SSRN website:
- from the RePEc website:
- from the CESifo website:
WWW.SSRN.com
www.RePEc.org
www.CESifo-group.org/wp

\section{CESifo}




\title{
Compliance Dynamics Generated by Social Interaction Rules
}

\begin{abstract}
We study compliance dynamics generated by a large set of behavioral rules describing social interaction in a population of agents facing an enforcement authority. When the authority adjusts the auditing probability every period, cycling in cheating-auditing occurs: Intensive monitoring induces compliance, but with high compliance there is incentive for lax monitoring; with less monitoring, compliance starts decreasing, and then there is an incentive to intensify monitoring. Thus, the real life phenomenon of compliance fluctuations is explained by the nature of social interaction process rather than by exogenous parameter shifts. For the authority committed to a fixed auditing probability, we derive a sufficient condition for fines to be effective means of deterrence. Our analysis can be applied, among others, to crime, tax evasion, safety regulations, employment and environmental protection.
\end{abstract}

JEL-Code: C790, D830, H260, K420.

Keywords: social interaction, compliance, random matching, behavioral rule, learning.

\author{
Vilen Lipatov \\ Department of Economics \\ Goethe University Frankfurt \\ Grüneburgplatz 1 \\ Germany - 60323 Frankfurt am Main \\ lipatov@em.uni-frankfurt.de
}

April 15, 2014

I am grateful to Ilya Gvozdevskiy, Marco Ottaviani, Joel van der Weele and participants of IIPF Congress Dresden and a seminar at Goethe University Frankfurt for discussion. I am also indebted to Chaim Fershtman and other seminar participants at Tel Aviv University for their valuable comments on an earlier version of this paper. 


\section{Introduction}

Compliance problems are pervasive in the life of mankind. Once a law is established, the temptation to break it is there as well. That is why compliance lies at the heart of the discipline of law and economics. Correspondingly, there is a very large body of literature that addresses the problem of compliance both from the perspective of the law and from the perspective of economics (and the intersection of these starting perhaps from Becker 1968).

Within this literature, there is a growing body of research that takes into account behavioral aspects ${ }^{1}$ of the compliance problem. In particular, social interactions feature prominently in such models (see van der Weele 2012 for a survey of relevant literature in economics of crime).

Most of the attention in this field was paid to indirect (or global) social interactions, i.e. social norms, intrinsic motivation, reputation. To complement these findings, we focus on direct (local) social interaction. Its analysis is exemplified by seminal articles of Sah (1991), who rolls out a general framework for analyzing crime dynamics with rational but uninformed agents, and of Glaeser at al (1996), who explain large cross-city variance of crime rate by imitation of the behavior of neighbors. In a series of controlled experiments, Cooper and Rege (2011) find direct social interactions to be the likely cause of peer group effects.

We theoretically contribute to the literature on direct social interactions by characterizing aggregate dynamics of compliance in large populations facing an enforcement authority, when each agent follows a behavioral rule rather than solves a complex maximization problem. A behavioral rule specifies the action in the current period conditioned on the own action in the previous period(s) and any information available about the others in the interaction group. Thus, instead of deriving behavior from preferences $^{2}$, we take (potentially observable) behavioral rules at individual level as given and derive population dynamics that they produce.

Our agents do not explicitly form any beliefs about auditing probability or population share of compliance - they simply follow their rule. Using survey data in the context of environmental regulation, Earnhart and Friesen (2013) reject the hypothesis that deterrence behavior is explained by standard rational models. To the contrary, they find evidence of strong influence of behavioral factors that they call "experiential deterrence" and that are reflected in our sample behavioral rules. At the same time, each behavioral rule could be rationalized by some preference relation and some belief updating procedure that does not necessarily follow Bayes' rule. There are both normative and positive reasons for considering non-Bayesian updating (Epstein et al 2010). The former is the cost of information processing that makes the simpler heuristic rules preferable over the complicated Bayesian ones; the latter is overwhelming experimental evidence suggesting that people do not use Bayesian rule in information processing (see e.g. Camerer 1995, Rabin 1998 for a survey; Grether

\footnotetext{
${ }^{1}$ See Bowles (1998) for a general account of endogenous preferences; Bar-Gill and Fershtman (2005) for the analysis of how public policy may shape preferences.

${ }^{2}$ There is a growing literature that incorporates behavioral aspects in compliance problems. For a survey on public economics experiments see e.g. Alm (2010).
} 
1980 for an early paper).

Our strategy is to identify a class of behavioral rules that generate similar population dynamics and then to characterize this dynamics. For this purpose, we consider only deterministic behavioral rules (stochastic rules naturally smoothen the evolution of behavior without changing our main findings) and we model social interaction as random matching. Random matching is extensively used in evolutionary game theory, general equilibrium theory, monetary and labor economics (see references in Duffie and Sun 2007); Kandori (1992) is one of the earlier papers that use random matching to analyze social interaction and "community enforcement". While putting more structure on the matching process, e.g. considering networks of agents as in e.g. Ballester et al. (2010), would certainly add realism to our model, it would also complicate it substantially. As considering behavioral rules is a novel approach to modeling compliance, we opt for a simplest formulation that allows to take into account direct social interaction.

The crucial ingredients in our setup are a single monitoring (controlling) authority and a large population of interacting agents that have an option to comply. These can be criminals vs. police, corrupted officials vs. anti-corruption body, firms not complying with quality or safety regulations vs. corresponding monitoring authorities, polluting producers vs. environmental authority, traffic violators vs. road police, free riders vs. controllers in public transportation, etc.

The main result of the model is the cycling dynamics of auditing and compliance generated by a class of rules that we call stabilizing. With myopic monitoring agency, both the share of noncompliant agents and the auditing intensity exhibit fluctuations giving rise to (stable) cycles. The system is cycling around a steady state, in which the share of cheaters is determined by the costs and benefits of the monitoring authority, whereas the auditing intensity is determined by the properties of the behavioral rule in question. In Appendix A9 we show that this result is robust to allow for infrequent rematching and multiperiod memory.

The intuition behind our cycling result is very simple and very robust. Since with a behavioral rule it takes time to react to a given strategy of monitoring authority, there will be a period of a decrease (increase) in compliance after auditing intensity reaches sufficiently low (high) level. The myopic behavior on the side of the authority leads, on the other hand, to adjustment of auditing intensity upwards (downwards) when the compliance level is low (high). For a large class of behavioral rules, we provide a simple sufficient condition for population dynamics to spiral to or away from steady state in continuous time.

According to an urban legend, a somewhat similar dynamics emerges in the number of students enrolled in classes - when there are few high quality students the average grade is high, attracting many lower-quality students next year when the average grade will be low so in the following year only highly motivated students will enroll, and so on. ${ }^{3}$ One can think of low quality students as noncompliant agents and the teacher as monitoring authority that sends a signal about the success probability in the course to future generations of students. In a more directly related to compli-

\footnotetext{
${ }^{3}$ I am grateful to Marco Ottaviani for this observation.
} 
ance context, public enforcement agencies usually have "action weeks" (or months, etc.) in which e.g. traffic violators or passengers without tickets are monitored more intensively. The existence of such periodicity may hint at the dynamic of our type whereby the authority is waiting for a certain threshold in the number of violations to trigger the "action time". ${ }^{4}$ Still another story consistent with our results comes from the recent European experience, whereby some countries (e.g. Greece) did not comply with fiscal rules because they were largely unmonitored by European authorities. Once these countries found themselves on the brink of a sovereign default, the monitoring got tightened and the widespread hope in Europe is that the compliance with EU rules in the problematic countries is rising. ${ }^{5}$ A similar story arises from the recent banking crisis experience: The banks were taking too much risk as a consequence of, among other factors, very loose monitoring in the banking sector. After the crisis, the monitoring activities increased substantially (e.g. creation of Single Supervisory Mechanism in Europe) and there is a lot of indirect evidence of banks becoming more compliant to prudent behavior.

\footnotetext{
${ }^{4}$ Consider, for instance, the following citation from the Road Safety Canada report: "Periodic Selective Traffic Enforcement Programs (STEPs) combine heightened police enforcement and public awareness campaigns and have been important in increasing belt use across the country. During the Canada Road Safety Week leading up to the May long weekend and Operation Impact during the Thanksgiving weekend in October, seat belt use is a major focus for enforcement campaigns by police services across the country." Available at https://www.tc.gc.ca/eng/motorvehiclesafety/tptp15145-1201.htm\#s313, accessed on 19.02.2014

${ }^{5}$ Thanks to Aitor Erce for pointing out this regularity.
} 


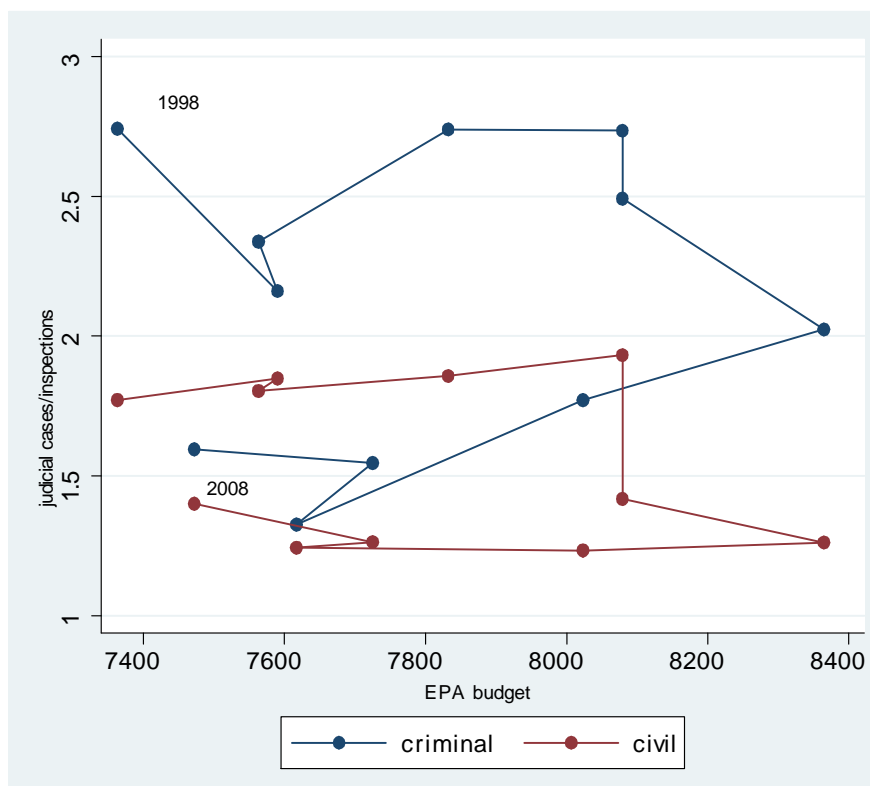

Figure 1. Environmental compliance/enforcement dynamics, 1998-2008. The data on EPA budget is from the Agency's website, http://www2.epa.gov/planandbudget/budget; accessed on 21.02.2014. The data on judicial cases and inspections is from Grey and Shimshack (2011).

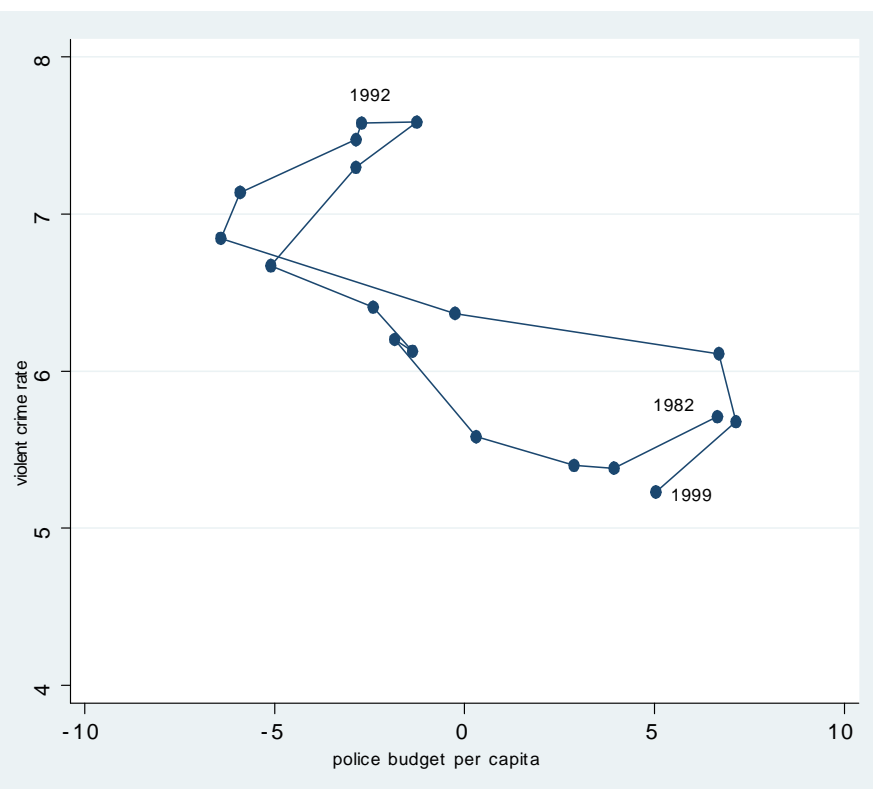

Figure 2. Crime/enforcement dynamics, 1982-1999.

The data on violent crime rate is from Uniform Crime Reporting statistics, the police budget per capita is in deviations from linear trend, the raw data is from the US Bureau of Justice,

http://www.bjs.gov/index.cfm?ty=tp\&tid=5, accessed on 21.2014 .

Consider the dynamics on figure 1. On the horizontal axis, we mark the US Environmental Protection Agency (EPA) budget as a proxy for our monitoring probability variable. On the vertical axis, we mark the ratio of judicial cases (civil or criminal) to inspections as a proxy of our non-compliance variable. The resulting dynamics are remarkably in line with our findings and, in particular, with the phase diagram on figure 3 presented in the section 2.3: Both noncompliance and enforcement grow first; then noncompliance goes down while enforcement still grows; then enforcement goes down, while noncomplience continues to decrease; finally, noncompliance increases while enforcement still decreases. A similar picture may be seen on figure 2, where we proxy non-compliance variable with violent crime rate in the US. We proxy the monitoring probability by the detrended per capita budget of the police (all levels). For the year 1992-95 this slightly deviates from our phase diagram, but otherwise it reflects the cyclical nature of the phenomenon as well. Clearly, these graphs do not empirically validate our results, as there is a multitude of other factors affecting compliance; the observed fluctuations are merely motivating for our analytical work.

With an authority committed to a constant over time auditing intensity, there is convergence to a steady state if the behavioral rule is stabilizing. We provide a simple sufficient condition for the fine to be an effective deterrent in such a steady state. Interestingly, the 'deterrence hypothesis' may fail in our setup because of the peculiarity of social interaction even if we assume that in the steady state higher auditing results in higher compliance. We thus introduce yet another reason (the social interaction effect) why the fines may be ineffective, complementing the explanations 
discussed in the literature so far (intrinsic motivation, signaling, competition - for details see van der Weele 2012). Further, we illustrate the applicability of our results with (i) a low information rule that only utilizes information about detection, and (ii) rules inspired by the literature on technology diffusion, namely contagion, social influence and social learning.

Our approach seems promising in a sense that it allows for simple characterization of complex behavior without complicated derivations of group-dependent utility functions ${ }^{6}$. Note that in order to formulate a behavioral rule we only need to observe the actual behavior. In contrast, rationalizing a rule would require structural estimation, which is in most cases prohibitive due to simultaneity and reflection biases, as outlined in Manski (2000). Instead, we may simply observe the rule and derive the aggregate behavior from it. This might allow us to make more accurate inference about the welfare effects of various policies in the context where it was not feasible so far.

An approach similar in spirit to ours is agent based modeling (e.g. Bloomquist 2006). The crucial difference is that we qualitatively characterize general properties of the dynamics generated by a class of rules, whereas agent base modeling extensively uses simulations to get quantitative results for a particular behavioral rule. An interesting related paper analyzing compliance dynamics in context of tax evasion is Davis et al (2003). They use a dual approach: In the analytical model, they assume particular forms of differential equations describing the aggregate dynamics; in the agent-based model, they generate the aggregate dynamics from individual behavior using simulations. This dual approach clearly illustrates the gap in the literature that we aim at filling: We derive the aggregate dynamics from individual behavior in a tractable analytical framework.

Agent-based modeling is often criticized on the ground that the scope of its results is not clear. Indeed, if the analysis is carried out for a particular behavioral rule, it is not obvious that the same results, or even in any way similar results, can be obtained for another rule, perhaps some rule that is in action in reality. We provide a solution to this problem: we show that if the rule picked up by an agent-based model falls into the class of rules identified here, the aggregate dynamics generated is qualitatively similar to the dynamics that would be generated by another rule from this class.

The rest of the paper is organized in the following way. Section 2 presents our framework of direct social interaction in compliance and states the general results about dynamics and comparative statics. Section 3 illustrates our results with a particular behavioral rule that we call "low information rule". Section 4 further illustrates the general result by applying our approach to the behavioral rules inspired by technology adoption literature. Limitations of the model and possible extensions are discussed in the concluding section.

\footnotetext{
${ }^{6}$ Benabou and Tirole (2011) convincingly argue that even taking account of "social preferences" does not suffice for explanation of actual behavior. They therefore develop a theory of moral behavior based on self-inference, which can be applied to the compliance problems. We follow a different, constructive approach: Instead of asking the question of what explains given behavior, we take potentially observable individual behavior as given and then derive the patterns of aggregate behavior from it.
} 


\section{The model}

\subsection{Repeated social interaction}

"[Many sociological] studies force us to start to kind of rethink our mental model of how we behave," said Duncan Watts, a Columbia University sociologist. "Public policy in general treats people as if they are sort of atomized individuals and puts policies in place to try to get them to stop smoking, eat right, start exercising or make better decisions about retirement, et cetera. What we see in this research is that we are missing a lot of what is happening if we think only that way." 7

We bring in social interaction to a simplest repeated (discrete time) setup with minimal behavioral assumptions. The problem can be formulated as follows. In period 0 the shares of non-compliant $q_{0} \in(0,1)$ and audited $p_{0} \in(0,1)$ agents in homogenous population (continuum of measure one) are given exogenously. Between period 0 and period 1 the agents are randomly matched in groups of $n$ and receive information specified by a given behavioral rule, potentially learning who got a better payoff. In period 1 the authority performs auditing that either maximizes its expected payoff in this period (no commitment) or maximizes its long-run payoff (commitment). The agents choose comply or not comply according to the behavioral rule. The "game" is then repeated infinitely, whereby the agents are rematched in every period (in Appendix A9 we consider possibility of no rematching).

In the present formulation the agents are not divided into groups or audit classes. Each may choose non-compliance, however, the authority has to prove the fact of non-compliance, even if it knows that non-compliance takes place. Such setup is especially appealing in application to corruption or free riding: a bureaucrat has to be caught receiving a bribe in order to be penalized, even if it is a common knowledge that she/he is corrupt; a passenger without a ticket will not be charged a fine unless she meets a controller.

Note that we cannot take a ready aggregate dynamics for the population because of the asymmetric nature of the players: in the no commitment case the authority is using myopic best response; the agents follow a behavioral rule. Without such asymmetry, our game resembles emulation dynamics as it is defined by Fudenberg and Levine (1998), which is known to converge to replicator dynamics under a number of assumptions including only two interacting agents. Our aggregate dynamics does not converge to replicator dynamics and has to be derived for each interaction rule.

\subsection{The setup and general results}

If the change in compliance depends on the state of the system in the previous period in a constant fashion, the evolution of the share of noncomplying agents can be generally represented as

$$
q_{t+1}=q_{t}+f\left(q_{t}, p_{t}\right)
$$

\footnotetext{
${ }^{7}$ Citation from "Social networks more powerful than thought?" by Rob Stein, washingtonpost.com, May 26, 2008
} 
where $t$ is time period. The function $f(q, p):[0,1]^{2} \rightarrow[-1,1]$ is determined by how a researcher models the compliance process. In our framework, this function is shaped by a behavioral rule. We define a behavioral rule for $n$ people meeting between periods as a mapping from a set of outcomes yesterday $X=$ \{compliant audited, compliant not audited, caught non-compliant, not caught non-compliant $\}^{n}$ into the set of actions today $Y=\{$ comply, not comply $\}$. We only consider deterministic rules, so this mapping is a function.

In Appendix A9 it is shown that under a behavioral rule the function $f$ is a polynomial of the order at most $n$ in each of the arguments, and hence continuous. ${ }^{8}$ We shall call the behavioral rules that imply $f_{q}(q, p)<0$ everywhere on the domain stabilizing, as the larger the share of noncompliance, the smaller the period-to-period increase in noncompliance (or the larger the decrease). Correspondingly, the rules with $f_{q}(q, p)>0$ everywhere will be called destabilizing. The rules with $f_{q}(q, p)=0$ will be called monotonic, as they imply monotonic dynamics for any feasible $p, q$. We do not study the rules that switch the sign of $f_{q}(q, p)$.

Define $\hat{q}(p):[0,1] \rightarrow[0,1]$ as a function that maps a set of possible auditing probabilities into a set of long-run outcomes of non-compliance shares, $\lim _{t \rightarrow \infty} q_{t}$, whenever it exists given $q_{0} \in(0,1)$. Define also $\bar{q}(p): \mathcal{P} \rightarrow(0,1)$ as a function that maps the set of auditing probabilities $\mathcal{P}$ into the set of interior steady state values of non-compliance shares. In other words, $\bar{q}(p)$ is an interior solution to the steady state condition: $f(\bar{q}(p), p)=0$. For both $\hat{q}(p), \bar{q}(p)$ to be single-valued, we need to restrict our attention to the rules that produce a unique interior steady state. This will be true for almost all 2-person rules, all 3-person imitation rules (the crucial feature of imitation rules is that the agents may not choose actions that they have not observed in the previous period(s)), and many other rules with arbitrary number of interacting people (examples of such rules may be found in Sections 3 and 4; for further examples see Lipatov 2003).

The following assumptions seem reasonable and will be kept for the rest of the paper:

Assumption 1. $\bar{q}^{\prime}(p)<0$.

Steady state noncompliance is decreasing in detection, as otherwise the punishment is not perceived as such by a behavioral rule. We do not want to consider the rules that imply that our agents enjoy the fines ${ }^{9}$.

\footnotetext{
${ }^{8}$ Our interpretation of behavioral rule as interactions within a group is not unique (we could think of an agent simply learning some information from $n-1$ other randomly drawn agents), but it gets particular appeal as we add possibility of no rematching in the Appendix A9. I am thankful to Sebastian Krautheim for this observation.

${ }^{9}$ There is a large literature in both criminology (Akers 1990, Doob and Webster 2003) and economics (Frey and Jegen 2001) explaining why the 'deterrence hypothesis' might not hold. Van der Weele (2012) gives three possible reasons for the potential failure of this hypothesis: (i) expressive law and informal sanctions; (ii) signaling 'tougher' type, (iii) promoting competition in illegal activities. The first reason is intimately related to the intrinsic versus extrinsic motivation discussed in e.g. Gneezy and Rusticcini (2000), whereby higher detection probability may crowd out voluntary or social-norm-induced compliance. We abstract from all such considerations in our model in order
} 
Lemma 1. Under Assumption 1, for stabilizing rules $f_{p}(q, p)<0$ everywhere on the domain; for destabilizing rules $f_{p}(q, p)>0$.

Proof. The proof is presented in Appendix A1.

The following proposition describes the period-to period evolution of the share of noncomplying agents.

Proposition 1. Consider the dynamics of the share of non-complying agents $q$ for a behavioral rule that satisfies the Assumption 1. For a stabilizing rule, if $p_{t} \in\left[0, \bar{q}^{-1}(1)\right]$, then $q_{t+1}>q_{t}$;

if $p_{t} \in\left[\bar{q}^{-1}(0), 1\right]$, then $q_{t+1}<q_{t}$; if $p_{t} \in\left(\bar{q}^{-1}(1), \bar{q}^{-1}(0)\right)$, then $q_{t}<\bar{q}\left(p_{t}\right) \Longrightarrow q_{t+1}>q_{t}, q_{t}>\bar{q}\left(p_{t}\right) \Longrightarrow q_{t+1}<q_{t}$. For a destabilizing rule the inequalities are reversed.

Proof. We shall prove the statement for the stabilizing rules, as it is completely analogous for the destabilizing ones. From (1) $q_{t+1}>q_{t} \Longleftrightarrow f\left(q_{t}, p_{t}\right)>0$. For an interior solution $\left(p_{t} \in\left(\bar{q}^{-1}(1), \bar{q}^{-1}(0)\right)\right)$ we have $q_{t}<\bar{q}\left(p_{t}\right) \Longrightarrow f\left(q_{t}, p_{t}\right)>$ $f\left(\bar{q}\left(p_{t}\right), p_{t}\right)=0$, since by Lemma $1 f_{q}(q, p)<0$ (the rule is stabilizing). Correspondingly, $q_{t}>\bar{q}\left(p_{t}\right) \Longrightarrow f\left(q_{t}, p_{t}\right)<0 \Longleftrightarrow q_{t+1}<q_{t}$. For a corner solution $\hat{q}(p) \in\{0,1\}$. As $f_{q}(q, p)<0$, either $f(0, p)<0$ and $q_{t+1}<q_{t}$, or $f(1, p)>0$ and $q_{t+1}>q_{t}$. Fix $q \in(0,1)$. For $p \mid \bar{q}^{-1}(1) \leq p<\bar{q}(q), f(q, p)>0$. By continuity $\exists \varepsilon>0 \mid f(q, p-\varepsilon)>0$. Now suppose $\exists p<\bar{q}^{-1}(1), q \mid f(q, p)<0$. Then $\exists p_{0}<\bar{q}^{-1}(1), q_{0} \mid f\left(q_{0}, p_{0}\right)=0$ that contradicts corner solution. Thus, $f(q, p)>0$. The proof for $p_{t} \in\left[\bar{q}^{-1}(0), 1\right]$ follows the same lines.

We can see that for stabilizing rules with small (large) values of auditing probability the non-compliance is increasing (decreasing). Unexpectedly, in the interval of auditing probability values that result in an interior solution, the change in the proportion of noncomplying agents is negatively related to their number. This "antiscale" effect is explained by the high enough detection probability, for which the caught noncomplying agents contribute more to the increase in the proportion of the compliant agents, than the compliant agents themselves.

Intuitively, Proposition 1 illustrates that stabilizing rules generate dynamics that conforms to the common wisdom of effective enforcement: If auditing is high, compliance increases in time, and visa versa. Interestingly, for destabilizing rules this intuition does not work, even though assumption 1 postulates the conventional relation (high enforcement - high compliance) in steady state. Paradoxically, the most sensible policy for the monitoring authority facing a destabilizing rule is to fix $p=0$ and let the noncompliance monotonically fall over time.

The following remark will allow us to get a better feeling about the dynamics generated by the rules:

to focus on the effect of direct social interactions. Interestingly, this does not preclude the possible failure of 'deterrence hypothesis' in our setup, as shown in section 2.3. 
Remark 1. Iff a behavioral rule is stabilizing and $\exists \bar{q}(p),|f(q, p)|<|q-\bar{q}(p)| \forall q$, then for a fixed detection probability $p$ the share of non-compliant agents $q$ monotonically converges to $\bar{q}(p)$.

Remark 2. If a behavioral rule is stabilizing and $\exists \bar{q}(p),|f(q, p)|>|f(q+f(q, p), p)| \forall q$, then for a fixed detection probability $p$ the share of non-compliant agents $q$ converges to $\bar{q}(p)$.

The latter remark is a familiar contraction mapping; both remarks say that if the dynamics do not jump too vividly from period to period, it has to exhibit some convergence.

As for the monitoring/enforcement authority, it maximizes its expected payoff for any given learning rule in the population by choosing an auditing intensity. Further we consider two cases for the behavior of the authority. If it is unable to announce its auditing probability and keep it forever, our dynamics have two dimensions: an already derived one for $q$ and another one for $p$. We start, however, with a simpler case, when the auditors can credibly commit to a certain constant in time strategy (probability), and hence the dynamics are collapsing to one dimension.

\subsection{Results: Commitment}

Assume that the authority commits to a certain auditing probability $p$ once and forever (this corresponds to the principle-agent framework in static games). This setup may seem unrealistic, but we have at least three reasons to consider it. Firstly, there is a well established tradition in the tax compliance literature (Andreoni et al 1998) that deals with committed tax authority. Secondly, if the authority could choose whether to commit or not, it would commit, as this allows for a weakly higher payoff. Thirdly, commitment models in the literature are usually criticized due to the standard result of full compliance of audited taxpayers. Such criticism does not apply in our framework, so our analysis of commitment case seems justified. ${ }^{10}$

Under commitment, the authority chooses $p$ to maximize its steady state payoff

$$
(1-\hat{q}(p)) V+p \hat{q}(p) F-c p .
$$

Here $V>0$ is the value of compliance for the authority, $F>0$ is the value from detection and punishment, and $c>0$ is marginal auditing cost, assumed constant (not dependent on $p$ ) for simplicity. The value of noncompliance is normalized to zero. What exactly we mean by value in this setup depends on the application and objective of the monitoring authority. For instance, suppose that the objective is maximizing social welfare and the application is environmental protection (say, firms are audited for the amount of emissions). Then the value of compliance is the social welfare attainable under lawful amount of pollution. The value of detection/punishment is the value of compliance plus the social benefit of public funds obtained from fines

\footnotetext{
${ }^{10}$ In the Apendix A10 we show that the results of this subsection still hold in steady state of the setting where the monitoring authority is maximizing the payoff over the whole dynamic path rather then the steady state payoff only.
} 
minus any social costs that a fine might impose, perhaps in the form of reduced trust in the society. The value of noncompliance is the social welfare attainable under unlimited pollution.

Further, we assume linear monitoring costs; values of compliance, noncompliance and detection are independent of pervasiveness of noncompliance and auditing probability. We do it for simplicity as we want to focus on the effect of social interactions.

The first order condition for an interior solution is

$$
\left(p^{*} \bar{q}^{\prime}\left(p^{*}\right)+\bar{q}\left(p^{*}\right)\right) F=\bar{q}^{\prime}\left(p^{*}\right) V+c .
$$

Here the difference between the two steady state non-compliance functions is illustrated. Whereas $\hat{q}(p)$ describes the steady state for any admissible value of auditing probability, $\bar{q}(p)$ only characterizes interior steady state, and thus excludes all the values of $p$ that result in full compliance or full disobedience. The condition (3) is a familiar equality of marginal expected benefit (left hand side) and marginal expected cost (right hand side) of auditing an additional agent. The second order condition is then

$$
d:=\left(p^{*} \bar{q}^{\prime \prime}\left(p^{*}\right)+2 \bar{q}^{\prime}\left(p^{*}\right)\right) F-\bar{q}^{\prime \prime}\left(p^{*}\right) V<0 .
$$

To sharpen presentation, we will further limit our attention to the case of no relation between the values of $V$ and $F$, i.e. when the following assumption is satisfied:

Assumption 2. The value of compliance $V$ and the value of punishment $F$ are independent, $V^{\prime}(F)=0$.

The comparative statics for the interior solution ${ }^{11}$ gives

$$
\begin{aligned}
& \frac{d p^{*}}{d c}=\frac{1}{d}<0, \\
& \frac{d p^{*}}{d F}=-\frac{p^{*} \bar{q}^{\prime}+\bar{q}}{d},
\end{aligned}
$$

where we use $\bar{q}$ and $\bar{q}^{\prime}$ to stand for the value of these functions at $p^{*}$ for the ease of exposition.

Higher auditing costs have unambiguously negative effect on steady state auditing probability and hence positive effect on steady state non-compliance (recall that $\bar{q}^{\prime}\left(p^{*}\right)<0$ by Assumption 1). The effect of the fine ${ }^{12}$ is ambiguous and depends on the detection-probability-elasticity of steady state noncompliance, $\varepsilon(p):=-\frac{\bar{q}^{\prime}(p)}{\bar{q}(p)} p$. If $\varepsilon\left(p^{*}\right)<1$, the 'deterrence hypothesis' is confirmed for independent values of punishment (fine) and compliance, that is higher fine increases compliance. However, if $\varepsilon\left(p^{*}\right)>1$, then increasing the fine may lead to a drop in compliance. The intuition is analogous to that in how a price change affects revenues depending on the demand

\footnotetext{
${ }^{11}$ Note that these conditions do not hold on the border.

${ }^{12}$ Strictly speaking, it is not granted that the effect of the fine has the same direction as the effect of the value of detection and punishment. However, for revenue maximizing authority it is true. It is also true for social welfare maximizing authority, if the increase in the benefit of public funds provided by the fine is larger than the increase in the social costs of this fine.
} 
elasticity. Indeed, from (2) the effect of a change in the value from detection and punishment on the objective function of the authority is, by envelope theorem, the share of agents caught cheating in steady state, $p^{*} \hat{q}\left(p^{*}\right)$. This share is increasing in auditing probability iff $\varepsilon\left(p^{*}\right)<1$. Increase in $F$ exerts upward pressure on $p \hat{q}(p)$ (detection and punishment is more attractive), and this is transferred into an increase in $p^{*}$, or, equivalently, decrease in $\bar{q}\left(p^{*}\right)$ iff the steady state noncompliance is inelastic.

To sum up, the following proposition characterizes the effect of a change in enforcement.

Proposition 2. With a committed monitoring/enforcement authority and assumptions 1,2 satisfied, $\frac{d p^{*}}{d F}>0$ if and only if $\varepsilon\left(p^{*}\right)<1$.

Proof The proposition follows directly from (5) under assumptions 1, 2.

The proposition actually refines the requirement for a behavioral rule to result in the 'normal' behavior. Abstracting from any potential relation between the value of compliance and the value of fine, it states that the detection-probability-elasticity of steady state noncompliance should be smaller than one, $\varepsilon\left(p^{*}\right)<1$, in order for the rule to support the effectiveness of the fines. Correspondingly, if $\varepsilon\left(p^{*}\right)=1$, changing severance of punishment has no effect on compliance; if $\varepsilon\left(p^{*}\right)>1$, stricter enforcement is detrimental for compliance.

Though the failure of "deterrence hypothesis" has recently been discussed in the literature extensively (see van der Weele 2012), our discussion indicates a novel channel through which enforcement may be rendered ineffective, namely social interaction of agents following simple behavioral rules.

Finally, a note on dynamics, though one-dimensional, is in order for the committed authority case. From Proposition 1 and a fixed detection probability the following corollary is immediate:

Corollary 1. For a stabilizing rule, the population dynamics is characterized by convergence to a steady state in case of committed authority.

This result is reminiscent of Sah's (1991) finding of the existence of locally stable steady states in a setting where rational agents learn about the probability to be punished for criminal behavior despite the fact that "past crime breeds future crime".

\subsection{Results: Myopic authority}

No commitment and myopic behavior may be good assumptions for the compliance setting for at least three reasons. Firstly, the resources for monitoring activity are allocated by self-interested politicians who usually have short-term populist goals. Second, monitoring authorities often have neither capacity nor information to look far in the future; hence, they exhibit myopic behavior. Third, even if the decision maker has a long-term goal in mind, the actual decisions are not usually based on a formal model, but contain a lot of "expert judgment" that is not free from human biases, including myopia. 
A myopic authority decides on the optimal auditing rule in every period, assuming that the distribution of the agents (compliant vs. noncomplying) has not changed from the last period: $E q_{t+1}=q_{t}$ (fictitious play). A justification of such assumption may be that the authority does not know the behavioral rule and hence cannot compute $f\left(p_{t}, q_{t}\right)$ to make a more accurate prediction about the share of cheaters in the next period. Alternatively, we could assume $E q_{t+1}=q_{t}+f\left(p_{t}, q_{t}\right)=q_{t+1}$, but that does not change anything below as long as the authority only makes one period decisions (by the time authority decides on $p_{t+1}, q_{t+1}$ is fixed by the previous decisions and the behavioral rule). Thus, the crucial for this setting is myopy of the authority and not its belief about the future state.

The expected payoff of the authority is determined by (2), where $\hat{q}(p)$ is substituted by $q_{t}$. Maximizing the expected payoff, we get the following best response strategy:

$$
B R\left(q_{t}\right)=\left\{\begin{array}{l}
0, \text { if } q_{t}<\mu ; \\
1, \text { if } q_{t}>\mu .
\end{array}\right.
$$

where $\mu$ is the level of compliance that induces switch of best response from zero to one or back:

$$
\mu=\frac{c}{F} \text {. }
$$

As the authority is very unlikely to jump from not auditing anybody to auditing everybody and back, we explicitly augment the choice of the enforcement agency with an inertia variable ${ }^{14}$ :

$$
p_{t+1}=\alpha B R\left(q_{t}\right)+(1-\alpha) p_{t}
$$

where $\alpha$ determines the speed of adjustment; $B R$ is the best response function, which is defined above as the payoff maximizing $p$ given the belief about the distribution of the agents. With $\alpha \rightarrow 1$, we are back to the case of jumping of the auditing probability from 0 to 1 ; with $\alpha \rightarrow 0$, the probability of audit stays very close to an initial level forever ${ }^{15}$.

Combining equations (6) and (8) allows us to summarize the period-to-period dynamics of detection probability in the following lemma:

Lemma 2. If the enforcement authority plays a myopic best response with inertia on a population of imperfectly compliant agents, then $p_{t+1}>p_{t}$, if $q_{t}<\mu$; $p_{t+1}<p_{t}$, if $q_{t}>\mu$.

\footnotetext{
${ }^{14}$ the inertia assumption is common in the literature on learning and evolution - see e.g. Fudenberg and Levine (1998, p. 31). Convex cost of auditing would have a similar smoothing effect (see Appendix A.3).

${ }^{15} \mathrm{We}$ can also consider $\alpha$ to be adaptive to the audit benefit-cost ratio: $\alpha=\bar{\alpha}\left(\frac{q}{\mu}\right)$. The ratio is increasing in $q$, so if $\bar{\alpha}($.$) is an increasing function, inertia gets less pronounced at higher values of$ non-compliance. If $\bar{\alpha}($.$) is a decreasing function, the opposite is true. In either case, the dynamic$ patterns do not change much from the case of $\alpha$ being a constant and can still be characterized by the same phase diagram.
} 
Intuitively, the myopic best response in our setup transforms into a simple rule for the authority: if the compliance is below some threshold, increase the auditing; if the compliance is below this threshold, decrease the auditing.

An interior steady state is described by the following pair:

$$
q^{s s}=\mu, p^{s s}=\bar{q}^{-1}(\mu) .
$$

In Appendix A11 we show that such a steady state is unstable. Instead, our system generates steady cycles that are stable, if the behavioral rule is stabilizing and $f_{q}>-2$. The dynamics of the system for any behavioral rule under consideration (stabilizing or destabilizing) is captured by the phase diagram (figure 3).

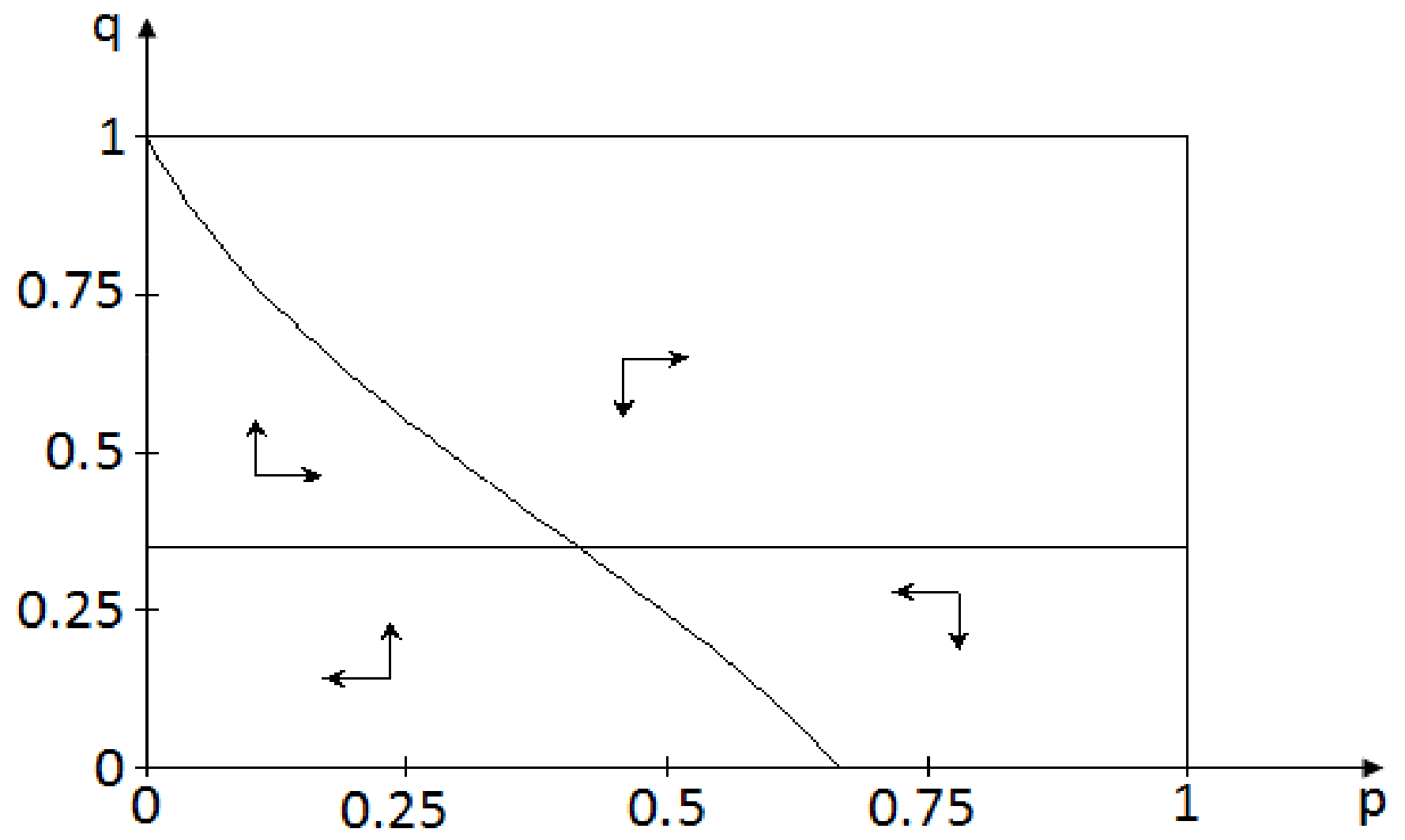

Figure 3. Phase diagram.

We can see that an interior steady state is the intersection of the horizontal constant auditing line $q=\mu_{1}$ and downward sloping constant compliance line $q=\bar{q}(p)$. These lines split the auditing-compliance simplex into 4 parts, which we can characterize with the help of Proposition 1 and Lemma 2. For a stabilizing rule, we have

- the south-west: non-compliance is increasing and auditing is decreasing.

- the north-west: non-compliance is increasing and auditing is increasing. 
- the north-east: non-compliance is decreasing and auditing is increasing.

- the south-east: non-compliance is decreasing and auditing is decreasing.

Intuitively, convergence a là remark 2 cannot be achieved in continuous time, if the detection probability is changing discretely. When the dynamics come too close to the steady state, a discrete shift pushes it away. For example, on the unit square with $p$ and $q$ dimensions, consider a point $(p, \mu)$ such that $p$ is sufficiently close to $p^{s s}$. From this point, the dynamic will jump to one of the points $(p, \mu \pm \Delta)$. But from this point, there is a discrete jump in auditing probability, which will overshoot $p^{s s}$. As a result, we can observe stable or unstable cycles.

To avoid the technical details presented in Appendix 11, we further focus on a continuous time approximation of our discrete time model. The following proposition shows that in continuous time the system converges to a steady state for a wide class of the behavioral rules.

Proposition 3. Consider a behavioral (learning) rule characterized by the transition function $q_{t+1}=q_{t}+f\left(q_{t}, p_{t}\right)$ with $\left|f_{i}\left(q_{t}, p_{t}\right)\right|<\infty, i=q, p$. With myopic authority, the interior steady state is stable in continuous time, if the learning rule is stabilizing, $f_{q}(q, p)<0$.

Proof. The proof is presented in Appendix A2.

Comparative static results for the steady state follow trivially from (9):

$$
\frac{d q^{s s}}{d c}>0, \frac{d q^{s s}}{d F}<0 .
$$

Correspondingly, under Assumption 1,

$$
\frac{d p^{s s}}{d c}<0, \frac{d p^{s s}}{d F}>0
$$

Thus, the auditing probability in steady state is decreasing in costs of auditing and increasing in the value of punishment.

Interestingly, the myopic authority does not seem to leave space for the nonconventional effect of tightening enforcement - indeed, $\frac{d q^{s s}}{d F}$ is unambiguously negative for any parameter values. Note though that the system may be far from steady state, and an increase in $F$ may either have very little effect on period-to-period compliance dynamics, or even result in a lower compliance in the next period. This is best illustrated on the phase diagram above.

To summarize our findings about the dynamics, we present the following corollary of Proposition 1 and Lemma 2:

Corollary 2. Consider a stabilizing behavioral rule that does not "jump" too much in the sense of remark 2. The population dynamics is characterized by stable clockwise cycles around a steady state in case of no commitment. 
Our result about cycles is remarkably in line with Cressman et al. (1998), who applied evolutionary dynamics to a game between asymmetric populations (criminals and home-owners). In their setup, the analogy with predator-prey dynamics is direct. We show that their result extends to many other situations, in which enforcement side cannot be modeled as a population, but rather as a (unable to commit) monitoring authority.

What horizon an enforcement authority has is a matter of a particular application. While many government agencies may have a natural inclination to short-term planning because of political cycle, non-governmental not-for-profit organizations, central banks and international bodies may have more long-term concerns.

\section{Example: low information rule}

\subsection{Description}

Suppose $m+1$ people meet (and $m$ is substantially larger than 2). Consider the following rule. For a not caught agent: if more than $k^{*}$ caught agents are observed, play comply in the next round, if less or equal - play not comply; for a caught agent: play comply. The intuition behind the rule is straightforward: an agent gets scared and chooses honesty, if she is caught, or if she observes more than $k^{*}$ of other caught agents.

This is a behavioral rule that requires minimal information about the individual behavior, and namely only whether he/she was caught not complying. We can assume that the availability of this information is assured by the monitoring authority for the purpose of deterring the others. This is beneficial for the monitor, if agents get scared observing somebody punished ${ }^{16}$. Since this rule does not require the knowledge of any characteristics of meeting agents, it can cover groups of substantial size, e.g. colleagues at a single firm or even the members of the same profession. Then the ease of information dissipation will be reflected in the group size in our model, and we can see how it influences compliance and auditing.

The probability to observe less or $k^{*}$ caught individuals is defined by cumulative binomial distribution function

$$
\begin{gathered}
\operatorname{Pr}\left(k \leq k^{*}\right)=\sum_{i=0}^{k^{*}} b(i, m, p q), \\
b(i, m, p q) \quad: \quad=\left(\begin{array}{c}
m \\
i
\end{array}\right)(p q)^{i}(1-p q)^{m-i} .
\end{gathered}
$$

Then cheating is evolving according to ${ }^{17}$

$$
q_{t+1}=\left(1-q_{t} p_{t}\right) \operatorname{Pr}\left(k \leq k^{*}\right) .
$$

\footnotetext{
${ }^{16}$ For the empirical motivation of this assumption, see Rincke and Traxler (2011) in the context of compliance with TV license fees in Austria; Galbiati and Zanella (2012) in the context of tax evasion in Italy. Both studies find that audits generate substantial social multipliers.

${ }^{17}$ The existence result for independent random matching is provided by Duffie and Sun (2007).
} 
Though the rule is stabilizing (we show that formally in Appendix A4), the problem with its dynamics is that once the system comes close to extreme values of $q$ ( 0 or 1), it is jumping between "almost all cheating" and "almost all honest" states in every period. This problem obviously stems from an 'epidemic' nature of the specified principle: once there are very many cheaters, almost everybody meets a caught cheater, and then all those switch to playing comply. But once almost everybody is playing comply, almost nobody meets a caught cheater, and then almost everybody is playing not comply. Proposition 3 still applies, as continuous time naturally smoothes the jumping described above.

The usual method to make the dynamics smoother is to introduce some kind of inertia into the system, just like it was already done from the side of the monitoring authority. So, let us say that with probability $\beta$ every unpunished agent changes his/her action according to already specified rule, and, correspondingly, with probability $1-\beta$ plays the same action as in the previous period. As before, punished agents switch to compliance with probability 1 , and thus do not exhibit any inertia.

Then in every period $(1-\beta)(1-p) q+\beta q(1-p) \operatorname{Pr}\left(k \leq k^{*}\right)$ cheaters remain cheaters plus $\beta(1-q) \operatorname{Pr}\left(k \leq k^{*}\right)$ compliant people switch to non-compliance. The dynamics is described by

$$
q_{t+1}=q_{t}\left(1-p_{t}\right)(1-\beta)+\beta\left(1-q_{t} p_{t}\right) \operatorname{Pr}\left(k \leq k^{*}\right) .
$$

For small enough values of $\beta$ it converges to a steady state (cycle in discrete time) rather than jumps between two extreme values. For simplicity we further consider the case when observing one caught individual is enough to deter from evasion $\left(k^{*}=0\right)$. The dynamics is then

$$
q_{t+1}=q_{t}\left(1-p_{t}\right)(1-\beta)+\beta\left(1-q_{t} p_{t}\right)^{m+1}
$$

\subsection{Properties}

As stated by corollary 2, with myopic authority we again observe cycles around the steady state. The position of steady-state noncompliance curves is determined by two factors: inertia in decision making $\beta$ and a number of people to meet $m$. Notice, however, that even for $p \rightarrow 1$ cheating is not eliminated completely. Indeed, for

$p_{t}=1, q_{t+1}=\beta\left(1-q_{t}\right)^{m+1}$, so that $q=0$ only for $\beta=0$, which is impossible. This seemingly strange result stems from the poor information the individuals possess: if nobody is cheating, nobody is caught, so in the next period $\beta$ of individuals will cheat.

Proposition 4. For low information rule with myopic monitoring authority, the steady state auditing is decreasing in $m$ and $\beta, \frac{d p^{s s}}{d m}<0, \frac{d p^{s s}}{d \beta}<0$.

The proof is presented in Appendix A5. Intuitively, the larger the interaction groups are $(m)$, the more precisely the number of occurrences in them reflects the population auditing, the less is actual steady state auditing that is required to maintain compliance rate of $c / F$. The size of interaction group can be also thought of as 
the speed of information dissipation: the faster information is spread in the population, the less effort is needed from the monitoring authority.

The smaller inertia $(\beta)$ also reduces the effort of the authority in the steady state. Again, smaller inertia means that new information is faster transferred into action, so that monitoring becomes a more efficient deterrent.

With committed forward-looking authority, noncompliance may decrease or increase over time depending on whether $q_{t}>\hat{q}\left(p^{*}\right)$ or the opposite, according to the Proposition 1. $\frac{d p^{*}}{d \beta}$ has an arbitrary sign. Increasing the number of people met brings about less auditing following the same intuition as in no commitment case. There is however an important limit to the applicability of this intuition to the commitment case. Namely, it may not work if the tax authority cares about punishment per se too much relative to compliance, i.e. if the value of compliance for the authority $V$ is smaller than the expected value of punishment $p^{*} F$. Formally, we have the following proposition:

Proposition 4a. For low information rule with committed monitoring authority, the steady state monitoring is decreasing in $m, \frac{d p^{*}}{d m}<0$, if $V>p^{*} F$ and $p^{*}$ is not too large.

The precise meaning of "not too large" is provided in the proof of proposition 4a, presented in Appendix A5.

\subsection{Rationalizability}

In the following we show that low information rule can be generated by utility maximization. Consider a rational model similar to that of Sah (1991), but taking into account some psychological features like limited memory, eigenbias and fear. Denote the payoff from compliance $V_{c}$, the payoff from non-compliance if not caught $V_{n}$ and the payoff from non-compliance if caught $V_{j}, V_{n}>V_{c}>V_{j}$. Then $u:=\left(V_{n}-V_{c}\right) /\left(V_{n}-V_{j}\right)$ is the "relative payoff to noncompliance". Analogously to Benabou and Tirole (2011) (of course, the context and the interpretation are completely different), define $v$ as the agent's perception about $p q$, the probability to be punished given that she is not compliant,

$$
v=\left\{\begin{array}{l}
v_{H} \quad \text { with probability } \rho \\
v_{L} \quad \text { with probability } 1-\rho
\end{array},\right.
$$

with $v_{H}>v_{L}$. Absent fear and limited memory, the agent will choose compliance iff $u \leq \mathbb{E} v$.

Denote the actual choice to comply or not in period $t$ by $a_{t}$, which takes the value 0 if non-compliant and value 1 if compliant. With probability $\beta$ unpunished agent remembers her belief $\rho$; with complementary probability $1-\beta$ she forgets her belief and simply plays the same action as in the previous period, $a_{t+1}=a_{t}$. The punished agent is scared for the next period and has a large (psychical) disutility of non-compliance, so that the only rational choice for her is to comply, $a_{t+1}=1$. A not 
punished agent who remembers her belief updates it conditioning on the last observed action of her own and those of her social interaction circle.

The trivial updating procedure rationalizing our rule is then $\rho_{t+1}=1$ if $k \leq k^{*}$ and $\rho_{t+1}=\rho_{t}$ otherwise, but any procedure of the type $\rho_{t+1}=\chi\left(\rho_{t}, k\right)$, where $\chi:[0,1] \times \mathbb{N} \rightarrow[0,1]$ is an increasing function of both arguments will do. Then, if we choose $\mathrm{u}$ in a way that $u<\mathbb{E} v$ for $\rho=1$ and $u>\mathbb{E} v$ for any $\rho: \rho<\bar{\rho}$ for some $\bar{\rho} \geq 0$, we are almost done. To complete the characterization, set $\rho_{0}<\bar{\rho}$, an expected-payoff-maximizing agent with evolution of beliefs described above chooses compliance either if she was caught or if she observed at least $k^{*}$ caught agents, in case she retains her memory. With the memory loss, she chooses compliance if and only if she chose it in the previous period. Thus, the payoffs and beliefs specified rationalize the low information behavioral rule.

Alternatively, if we want to stick to Bayesian updating, assume that agents who remember do so about their prior belief $\rho$ only, but not about their posterior belief. Then in each period their posterior subjective probability to be punished depends on the number of observed punished people $k$ in the following way:

$$
v=\left\{\begin{array}{ll}
v_{H} & \text { with probability } \frac{\rho b\left(k, m, v_{H}\right)}{\rho b\left(k, m, v_{H}\right)+(1-\rho) b\left(k, m, v_{L}\right)} \\
v_{L} & \text { with probability } \frac{(1-\rho) b\left(k, m, v_{L}\right)}{\rho b\left(k, m, v_{H}\right)+(1-\rho) b\left(k, m, v_{L}\right)}
\end{array} .\right.
$$

Clearly then

$$
\mathbb{E} v=\frac{v_{H} \rho b\left(k, m, v_{H}\right)+v_{L}(1-\rho) b\left(k, m, v_{L}\right)}{\rho b\left(k, m, v_{H}\right)+(1-\rho) b\left(k, m, v_{L}\right)} .
$$

In Appendix A6 we show that this is motonically increasing in $k$ from $v_{\text {min }} \geq 0$ to $v_{\max } \leq 1$. Hence, for any $0<k^{*} \leq m$ we can specify $u, v_{L}$ and $v_{H}$ in such a way that $u<\mathbb{E} v\left(k^{*}\right)$ and $u>\mathbb{E} v\left(k^{*}-1\right)$. Thus our payoffs and beliefs, updated every time from the same prior in Bayesian fashion, rationalize the low informational rule.

\section{Relation to technology adoption theories}

Among others, availability of concealment technology may serve as a reason for noncompliance. Indeed, in some contexts (tax avoidance being a leading example ${ }^{18}$ ) blunt non-compliance may be detected immediately. To have a considerable chance of non-detection, the agents need access to concealment expertise. In such cases, our behavioral rule may have a lot in common with the technology adoption process, whereby agents adopt the non-compliance strategy for some reason.

Young (2009) analyzes population dynamics generated by three classes of innovation diffusion theories: contagion, social influence and social learning. In this section we specify behavioral rules motivated by each of these theories. Due to specificity of compliance problem, we have to allow that there may be switches and reversal in the strategy to be adopted ${ }^{19}$. We show then that population dynamics generated by these rules in our context exhibits the patterns predicted by our model.

\footnotetext{
${ }^{18}$ See Lipatov (2012) for the analysis of situations in which concealment technology is provided by a distinct entity.

${ }^{19}$ The notion that the strategy to adopt may be changing over time (and indeed reversed) is not
} 


\subsection{Contagion}

The idea of contagion comes from the literature on epidemics: people adopt a new strategy simply by coming in contact with those who followed this strategy. In our context, we have to make an assumption on which strategy is contagious. One way to do it is to say that if the auditing probability is above some threshold, compliance is imitated; otherwise non-compliance is contagious. Then only compliant and noncomplying are relevant types, and the following table summarizes the shares of groups with $k$ compliant agents in the population together with their choices for the next period:

\begin{tabular}{|l|l|l|l|}
\hline types met & share & contagion if $p<\bar{p}$ & contagion if $p>\bar{p}$ \\
\hline all compliant & $(1-q)^{n}$ & compliant & compliant \\
\hline$k$ compliant & $\left(\begin{array}{l}n \\
k\end{array}\right)(1-q)^{k} q^{n-k}$ & $\lambda k$ become noncompliant & $\lambda(n-k)$ become compliant \\
\hline all noncompliant & $q^{n}$ & noncompliant & noncompliant \\
\hline
\end{tabular}

The period-to period dynamics is then

$$
q_{t+1}=\left\{\begin{array}{cl}
\sum_{k=0}^{n-1}\left(\begin{array}{l}
n \\
k
\end{array}\right)(1-q)^{k} q^{n-k} \frac{\lambda k+n-k}{n}, & \text { if } p<\bar{p} ; \\
1-\sum_{k=1}^{n}\left(\begin{array}{l}
n \\
k
\end{array}\right)(1-q)^{k} q^{n-k} \frac{\lambda(n-k)+k}{n}, & \text { if } p>\bar{p} .
\end{array}\right.
$$

In the Appendix A7 we show that this implies

$$
f_{q}(q, p)=\left\{\begin{array}{cc}
-\lambda\left(1-n t^{n-1}\right), & \text { if } p<\bar{p} \\
\lambda\left(n(1-t)^{n-1}-1\right), & \text { if } p>\bar{p}
\end{array}\right.
$$

Despite the fact that this contagion rule is converging to stabilizing only in the limit of large $n$ (for finite $n, f_{q}(q, p)<0$ only for $q \in\left(n^{-\frac{1}{n-1}}, 1-n^{-\frac{1}{n-1}}\right)$ ), the dynamics generated has the same patterns as outlined by Propositions 1, 3 for stabilizing rules. Namely, just like in Proposition 1,

- if $p_{t} \in[0, \bar{p}]$, then $q_{t+1}>q_{t}$;

- if $p_{t} \in[\bar{p}, 1]$, then $q_{t+1}<q_{t}$.

Proposition 3 also applies as long as $q^{S S} \in\left(n^{-\frac{1}{n-1}}, 1-n^{-\frac{1}{n-1}}\right)$, because stability property of the rule is used in its proof only locally, around steady state. The intuition about discrete time dynamics is also intact: for low auditing probability non-compliance is contagious, so the share of noncomplying agents monotonically increases. Once this share reaches $\mu$, auditing probability starts increasing, and once the auditing hits $\bar{p}$, compliance becomes contagious, so the share of non-compliant agents starts shrinking. Thus, we observe cycles described by Corollary 2 .

new to the literature on adoption. For a recent example, see Buera et al (2011), where governments learn whether market-oriented or interventionist strategy is preferable. 
A variation of this could be that whereas non-compliance needs special knowledge and therefore is slow to disseminate, the compliant behavior may spread instantaneously ${ }^{20}$. Then corresponding part of the law of motion is simply

$$
q_{t+1}=q^{n}, \text { if } p>\bar{p} .
$$

Thus, all the properties described above are preserved, only compliance is now faster to react to sufficiently high auditing probability.

\subsection{Social influence}

The idea behind social influence is that of popularity. The decision to adopt noncompliance is then based not on how good or desirable it has proven to be, but rather on how many peers have adopted it. There exist a threshold $k^{*} \geq 0$ (called the social threshold) such that the agents adopt (with probability $\lambda$ ) as soon as $k^{*}$ or more agents in the group have already adopted. This is similar to our low information rule, only applied not to the event of punishment, but to the compliance/noncompliance decision. Whereas in some applications (e.g. crime) it is reasonable to assume that the information about punishment, but not about undetected non-compliance, is available, in other applications (e.g. violation of traffic rules) it is reasonable to assume that punishment is not observed in the group, but compliance choice is known.

We again have compliant/non-compliant as relevant types and

\begin{tabular}{|l|l|l|l|}
\hline types met & share & influence if $p<\bar{p}$ & influence if $p>\bar{p}$ \\
\hline all compliant & $(1-q)^{n}$ & compliant & compliant \\
\hline$k<k^{*},\left(n-k<k^{*}\right)$ & $\left(\begin{array}{l}n \\
k\end{array}\right)(1-q)^{k} q^{n-k}$ & no change & no change \\
\hline$k>k^{*},\left(n-k>k^{*}\right)$ & $-"-$ & $\lambda k$ become noncompliant & $\lambda(n-k)$ become compliant \\
\hline all noncompliant & $q^{n}$ & noncompliant & noncompliant \\
\hline
\end{tabular}

The period-to period dynamics is then

$q_{t+1}=\left\{\begin{array}{cl}\sum_{k=0}^{k^{*}-1}\left(\begin{array}{l}n \\ k\end{array}\right)(1-q)^{k} q^{n-k} \frac{n-k}{n}+\sum_{k=k^{*}}^{n-1}\left(\begin{array}{l}n \\ k\end{array}\right)(1-q)^{k} q^{n-k} \frac{\lambda k+n-k}{n}, & \text { if } p<\bar{p} ; \\ 1-\sum_{k=n-k^{*}+1}^{n}\left(\begin{array}{l}n \\ k\end{array}\right)(1-q)^{k} q^{n-k} \frac{k}{n}-\sum_{k=1}^{n-k^{*}}\left(\begin{array}{l}n \\ k\end{array}\right)(1-q)^{k} q^{n-k} \frac{\lambda(n-k)+k}{n}, & \text { if } p>\bar{p} .\end{array}\right.$

Clearly, for $k^{*}=1$ we are back to the contagion rule. There is no qualitative change in the dynamics. Since we simply change the weights on some of base Bernstein polynomials, different thresholds span a family of rules ranging from contagion to status quo. The latter is another extreme, whereby social threshold is all agents in a group, $k^{*}=n$ :

$$
f(q, p)=\left\{\begin{array}{cc}
\sum_{k=0}^{n-1} B_{k, n}(t) \frac{n-k}{n}-1+t, & \text { if } p<\bar{p} \\
t-\sum_{k=1}^{n} B_{k, n}(t) \frac{k}{n}, & \text { if } p>\bar{p}
\end{array}\right.
$$

\footnotetext{
${ }^{20}$ Harris and Lopez-Valcarcel (2008) provide empirical evidence from cigarette smoking that the social influence may be asymmetric. In their study, a smoking sibling raises the probability to smoke significantly more than a non-smoking sibling lowers it. Mocan and Bali (2010) find evidence of asymmetric response of crime to changes in the unemployment rate.
} 
In other words, $f(q, p)=0$. With higher $k^{*}, f(q, p)$ becomes smaller by absolute value at every point in the domain up to the limit in which it is zero. Thus, with the social influence rule, we will see slower convergence and smaller cycles than with the contagion rule, otherwise the pattern remains the same.

\subsection{Social learning}

Social learning refers to a vast family of models that assume that the decision to adopt follows from rational evaluation of evidence about costs and benefits of the adoption. The assumptions of such models vary a lot, but there is considerable empirical evidence that learning from the experience of others does occur ${ }^{21}$. Following the logic of social leaning models, we assume that the decision to switch to compliance/noncompliance is made judging by its expected payoff.

In a little abuse of notation, denote compliance payoff $V_{c}$; non-compliance payoff in case not detected $V_{n}$; non-compliance payoff in case detected zero (normalized), $0<V_{c}<V_{n}$. In case the detection probability $p$ were known, the uncomplicated (without self-image or other-regarding concern) agents would simply choose compliance whenever $V_{c}>(1-p) V_{n}$. If the probability is unknown, an agent forms a belief $v$ about it as described by (13) with $v_{H}>v_{L}$ and expectation being $\bar{v}=\rho v_{H}+(1-\rho) v_{L}$. Then, an agent chooses compliance whenever $V_{c}>(1-\bar{v}) V_{n}$.

The belief may be formed in many ways, using any information available about the members of the group. One simple case, which is in a sense the opposite to contagion and social influence, is when the information about compliance/non-compliance is not used, and only information about monitoring is available. Namely, each agent knows the proportion of the monitored agents in his group and uses it as a belief about the probability of auditing. This is not interesting - all dynamics depend on $p$ only.

Instead, consider a rule according to which everybody forgets their previous beliefs: those who were non-compliant think that they believed that $v=v_{L}$ with probability 1 and those who were compliant think that they believed $v=v_{L}$ with probability 1 . To update this belief, an agent uses the number of audits in her group. The updating procedure can be specified so that for sufficiently small number or a sufficiently large number of audits everybody is convinced to switch to non-compliance or compliance, correspondingly. For some number of audits in a group, the update may result in a status quo.

The number of non-compliant in a group is independent of the number of audited in this group because of random auditing assumption. Thus, the population shares of these groups may be formulated as in the following table ( $l$ is the number of audited

\footnotetext{
${ }^{21}$ For the references to both theoretical and empirical literature on social learning see Young (2009), p. 1909. A curious recent piece of evidence directly relevant to compliance is learning to use steroids in baseball, analyzed in Gould and Kaplan (2011). They also find that the learning effect disappears after the introduction of drug testing.
} 
agents in the group):

\begin{tabular}{|l|l|l|}
\hline types met & share & not comply \\
\hline all not audited & $(1-p)^{n}$ & all \\
\hline$l \leq l_{1}$ & $\left(\begin{array}{l}n \\
k\end{array}\right)(1-q)^{k} q^{n-k}\left(\begin{array}{l}n \\
l\end{array}\right)(1-p)^{n-l} p^{l}$ & all \\
\hline$l_{1}<l<l_{2}$ & $-"-$ & $\frac{n-k}{n}$ \\
\hline$l \geq l_{2}$ & $-"-$ & none \\
\hline all audited & $p^{n}$ & none \\
\hline
\end{tabular}

Here, $l_{1}$ is the maximal number of audited agents in a group that induces a previously compliant agent to switch to non-compliance; $l_{2}$ is the minimal number of audited agents in a group that induces a previously noncomplying agent to switch to compliance. The following example of a simple belief updating procedure can rationalize such behavior: the previously compliant update their belief to $\rho=(1+l / n) / 2$; the previously non-compliant update their belief to $\rho=l /(2 n)$ (simple average of prior and the share of audited agents in the group).

In Appendix A8 we provide explicit expressions for the thresholds $l_{1}$ and $l_{2}$. We also show that the derivative we are interested in is

$$
f_{q}(q, p)=\sum_{l=l_{1}+1}^{l_{2}-1}\left(\begin{array}{l}
n \\
l
\end{array}\right)(1-p)^{n-l} p^{l}-1 .
$$

Thus, our social learning rule is stabilizing. If also assumption 1 is satisfied, then all our propositions and corollaries apply in this case. In the appendix we show that the thresholds can be chosen in a way to satisfy assumption 1.

\section{Conclusion}

The model presented in the paper is designed to capture a number of features of reality, which were underrepresented in the literature on compliance. These features are social interaction, poor knowledge of auditing probability, asymmetry in the behavior of two parties under consideration (population of agents vs. single authority), and intertemporal nature of the compliance decision. The interaction in the model is within groups to which individuals are assigned by random matching. Once in the group, a specific type of the individual (her compliance and detection history) and the types of other group members determine which action will be taken in the next period. Thus, agents follow some behavioral rule without solving complicated maximization problems.

The model allows us to identify a class of behavioral rules that result in cycling compliance-auditing dynamics, if the authority is not able to commit. When commitment to a fixed auditing probability is an option, we identify the class of rules for which compliance converges to an interior steady state. In such a steady state, fines may not be an effective deterrence simply because of social interaction, and not necessarily for other reasons discussed in the literature so far. 
Our model is a first step towards providing theoretical background for agent-based modeling and solid microfoundations for assumptions on aggregate population dynamics. Clearly, further research is needed to incorporate heterogeneous population, various auditing classes and non-random group formation in our model. Aliprantis et al (2007) consider a heterogeneous population in the framework with random pairwise matching (into groups of size $n=2$ ). To the best of our knowledge, no results are available so far for groups of larger size.

In general, the social interaction approach to compliance with simple behavioral rules reopens a whole bunch of policy issues. Are the recommendations of equilibrium theory valid, if the system does not converge to equilibrium? Are some changes in the existing policy worth undertaking, if we take into consideration not only difference in benefits between initial and final states, but also the costs of transition? Can the decision rules of the authorities and the learning mechanisms governing agents' behavior be manipulated in the way to achieve maximal social welfare?

As a building block for more general models, the behavioral approach can be employed in the studies on how the government can ensure a higher degree of trust in society (and more compliance as a result), how it can provide optimal (from the point of view of social welfare) level of public goods, how it can bring about faster growth of an economy. We leave these ambitious and exciting questions as an avenue for further research.

\section{References}

[1] Akers R. L. 1990. "Rational choice, deterrence, and social learning theory in criminology: the path not taken." Journal of Criminal Law and Criminology, 81: 65376 .

[2] Aliprantis C., Camera G. and Puzzello D. 2007. "A Random Matching Theory." Games and Economic Behavior 59, 1-16.

[3] Alm J. 2010. "Testing Behavioral Public Economic Theories in the Laboratory." National Tax Journal, 63, 635-658.

[4] Andreoni J., Erard J. and Feinstein J. 1998. "Tax Compliance." Journal of Economic Literature, 36(2), 818-860.

[5] Ballester C., Calvo-Armengol A., and Zenou, Y. 2010. "Delinquent Networks." Journal of the European Economic Association, 8(1), 34-61

[6] Bar-Gill O. and Fershtman C. 2005. "Public Policy with Endogenous Preferences." Journal of Public Economic Theory, 7(5), 841-857.

[7] Becker G. 1968. "Crime and Punishment: an Economic Approach." Journal of Political Economy, 76, 169-217.

[8] Benabou R. and J. Tirole. 2011. "Identity, Morals, and Taboos: Beliefs as Assets." The Quarterly Journal of Economics 126, 805-855. 
[9] Bloomquist K. 2006. "A Comparison of Agent-Based Models of Income Tax Evasion." Social Science Computer Review, 24(4), 411-425.

[10] Bowles S. 1998. "Endogenous Preferences: The Cultural Consequences of Markets and other Economic Institutions." Journal of Economic Literature, 36(1), 75-111.

[11] Buera F., A. Monge-Naranjo, and G. Primiceri. 2011. "Learning the Wealth of Nations." Econometrica, 79(1), 1-45.

[12] Camerer C. 1995. "Individual decision-making," in Handbook of Experimental Economics, J. Kagel and A. Roth eds., Princeton U. Press.

[13] Cooper D. and Rege M. 2011. "Misery loves company: Social regret and social interaction effects in choices under risk and uncertainty." Games and Economic Behavior 73, 91-110

[14] Cressman R., Morrison W. and Wen J-F. 1998. "On the Evolutionary Dynamics of Crime." Canadian Journal of Economics, 31(5), 1101-1117.

[15] Davis J., Hecht G., and Perkins J. 2003. "Social Behaviors, Enforcement, and Tax Compliance Dynamics." The Accounting Review, 78(1), 39-69.

[16] Doob Anthony N. and Cheryl Marie Webster. 2003. "Sentence Severity and Crime: Accepting the Null Hypothesis." Crime and Justice, 30, 143-195.

[17] Duffie D. and Sun Y. 2007. "Existence of Independent Random Matching." The Annals of Applied Probability, 17(1), 386-419.

[18] Earnhart D. and Friesen L. 2013. "Can Punishment Generate Specific Deterrence Without Updating? Analysis of a Stated Choice Scenario," Environmental and Resource Economics 56, 379-397.

[19] Epstein L., Noor J., and Sandroni A. 2010. "Non-Bayesian Learning," The B.E. Journal of Theoretical Economics, 10(1), 1 (Advances).

[20] Frey, B. and Jegen R. 2001. "Motivation Crowding Theory". Journal of Economic Surveys, 15(5), pp. 589-611.

[21] Fudenberg D.and Levine D. 1998. The theory of learning in games. MIT Press.

[22] Galbiati R. and Zanella G. 2012. "The Tax Evasion Social Multiplier: Evidence from Italy." Journal of Public Economics 96(5-6), 485-494.

[23] Glaeser E, Sacerdote B, and Scheinkman J. 1996. "Crime and social interactions". The Quarterly Journal of Economics, 111(2), 507-548.

[24] Gneezy, U. and Rustichini, A. 2000. "Pay Enough or Don't Pay at All". The Quarterly Journal of Economics, 115(3), 791-810. 
[25] Gould E. and Kaplan T. 2011. "Learning unethical practices from a co-worker: The peer effect of Jose Canseco." Labour Economics, 18(3), 338-348.

[26] Graetz M., Reinganum J. and Wilde L. 1986. The Tax Compliance Game: Towards an Interactive Theory of Law Enforcement. Journal of Law, Economics and Organization, 2(1), 1-32.

[27] Gray W. and Shimshack, J. 2011. The Effectiveness of Environmental Monitoring and Enforcement: A Review of the Empirical Evidence. Review of Environmental Economics and Policy, 5(1), 3-24

[28] Grether, D. 1980. "Bayes Rule as a Descriptive Model: The Representativeness Heuristic." The Quarterly Journal of Economics, 537-558.

[29] Harris J. and Lopez-Valcarcel B. 2008. "Asymmetric peer effects in the analysis of cigarette smoking among young people in the United States, 1992-1999." Journal of Health Economics 27, 249-264.

[30] Hirsch M. and Smale S. 1974. Differential equations, dynamical systems, and linear algebra. New York: Academic Press.

[31] Kandori M. 1992. "Social Norms and Community Enforcement." The Review of Economic Studies, 59(1), 63-80.

[32] Lipatov V. 2003. "Evolution of Tax Evasion." MPRA Paper 966.

[33] Lipatov V. 2012. "Corporate Tax Evasion: The Case for Specialists." Journal of Economic Behavior and Organization 81(1), 185-206.

[34] Manski C. 2000. "Economic Analysis of Social Interactions." Journal of Economic Prospectives, 14(3), 115-136.

[35] Mocan H. and Bali T. 2010. "Asymmetric Crime Cycles." The Review of Economics and Statistics 92(4), 899-911.

[36] Rabin M. 1998. "Psychology and economics". Journal of Economic Literature $36(1), 11-46$.

[37] Rincke J. and Traxler C. 2011. "Enforcement Spillovers." The Review of Economics and Statistics 93(4), 1224-1234.

[38] Sah R. 1991. "Social Osmosis and Patterns of Crime." Journal of Political Economy, 99(6), 1272-1295.

[39] Shimshack J. 2009. "Monitoring, Enforcement, \& Environmental Compliance: Understanding Specific \& General Deterrence." A Report Prepared for the Environmental Protection Agency's Office of Enforcement and Compliance Assurance (OECA). 
[40] Uniform Crime Reporting Statistics. 2012. Available at http://www.ucrdatatool.gov/

[41] Van der Weele, Joel J. 2012. "Beyond the State of Nature: Introducing Social Interactions in the Economic Model of Crime." Review of Law ES Economics, 8:1, 401-32.

[42] Young, H. 2009. "Innovation Diffusion in Heterogeneous Populations: Contagion, Social Influence, and Social Learning." American Economic Review, 99(5), 1899 1924

\section{Appendix}

\section{A1. Proof of Lemma 1}

By implicit function theorem, $f_{p}(\bar{q}(p), p)=-\bar{q}^{\prime}(p) f_{q}(\bar{q}(p), p)$. Under Assumption 1 for stabilizing rules then $f_{p}(\bar{q}(p), p)<0$. Suppose $\exists q \mid f_{p}(q, p)>0$. By continuity (a derivative of a polynomial is again a polynomial and hence continuous), $\exists q_{0} \in$ $(q, \bar{q}(p)) \mid f_{p}\left(q_{0}, p\right)=0$, which contradicts the uniqueness of the steady state. This proves the lemma for $p \in\left(\bar{q}^{-1}(1), \bar{q}^{-1}(0)\right)$. For $p \in\left[0, \bar{q}^{-1}(1)\right]^{22}$, by continuity in both arguments $\exists \delta, \varepsilon>0 \mid f_{p}\left(1-\delta, \bar{q}^{-1}(1)-\varepsilon\right)<0$. Suppose $\exists p, q \mid f_{p}(q, p)>0$, then again by continuity $\exists q_{0} \in(q, 1-\delta), p_{0} \in\left(p, \bar{q}^{-1}(1)-\varepsilon\right) \mid f_{p}\left(q_{0}, p_{0}\right)=0$. But this would mean that $q_{0}, p_{0}$ constitute a steady state, that contradicts the corner solution. The proof for $p \in\left[\bar{q}^{-1}(0), 1\right]$ is completely analogous. For destabilizing rules $f_{p}(\bar{q}(p), p)>0$ and the rest of the proof follows the same logic.

\section{A2. Proof of Proposition 3}

To investigate stability of the steady state analytically, we have to make two approximations. First, consider the system in continuous time: this makes sense, if we imagine that both the monitoring authority and individuals update their compliance and auditing decisions every day, rather than fixing it once for a whole year. We can rewrite our system of equations as

$$
\begin{aligned}
& q_{\tau+\Delta}=q_{\tau}+\Delta f\left(q_{\tau}, p_{\tau}\right), \\
& p_{\tau+\Delta}=p_{\tau}+\Delta g\left(q_{\tau}, p_{\tau}\right)
\end{aligned}
$$

and letting $\Delta$ be very small $\left(\frac{1}{365}\right.$, if we think of daily updating), in the limit we obtain

$$
\begin{aligned}
& \dot{q}=f(q, p), \\
& \dot{p}=g(q, p)
\end{aligned}
$$

\footnotetext{
${ }^{22}$ In case $\left[0, \bar{q}^{-1}(1)\right]$ is not an empty set.
} 
where $f(q, p)$ is defined by the learning rule and $g(q, p)=\alpha(B R(q)-p)$.

The stability matrix of this system is $\left(\begin{array}{ll}\frac{\partial \dot{q}}{\partial q} & \frac{\partial \dot{q}}{\partial p} \\ \frac{\partial \dot{p}}{\partial q} & \frac{\partial \dot{p}}{\partial p}\end{array}\right)=\left(\begin{array}{ll}a_{11} & a_{12} \\ a_{21} & a_{22}\end{array}\right)$

$=\left(\begin{array}{cc}f_{q}(q, p) & f_{p}(q, p) \\ \alpha B R^{\prime}(q) & -\alpha\end{array}\right)$.

The problem with this formulation is that the best response function is not continuous at the point of steady state, so we cannot compute $B R^{\prime}\left(q_{s s}\right)$. To go around it, we can make the second approximation: instead of the discontinuous best response we take a continuous function $A B R(q)=\Phi\left(\frac{\bar{c}(q)-c}{\sigma}\right)$, which approaches $B R(q)=$ $\left\{\begin{array}{l}0, \text { if } \bar{c}<c \\ 1, \text { if } \bar{c}>c\end{array}\right.$ with $\sigma \rightarrow 0$. Conventionally, $\Phi$ is cumulative distribution function of a standard normal random variable. Then $A B R^{\prime}(q)=\phi\left(\frac{\bar{c}(q)-c}{\sigma}\right) \frac{\bar{c}^{\prime}(q)}{\sigma}$. Recalling the expression for $\bar{c}(q)$ and evaluating at steady state $\left(\bar{c}\left(q_{s s}\right)=c\right)$, we get

$$
A B R^{\prime}\left(q_{s s}\right)=\phi(0) \frac{F}{\sigma} \Rightarrow a_{21} \approx \frac{\alpha F}{\sqrt{2 \pi} \sigma^{2}} .
$$

Note that we can make $a_{21}$ (since it is positive) arbitrary large by making $\sigma$ small enough and thus getting better approximation of initial best response function.

Now we are ready to address the question of stability of the steady state. If the real parts of both eigenvalues of the stability matrix are negative, the steady state is stable (see, for example, Hirsch and Smale (1974). The eigenvalues of our system are

$$
\lambda_{1,2}=\frac{1}{2}\left(a_{11}+a_{22} \pm \sqrt{\left(a_{11}-a_{22}\right)^{2}+4 a_{12} a_{21}}\right) .
$$

Note that by Lemma 1 in steady state $\frac{a_{12}}{a_{11}}>0$. Then, quite intuitively, the stabilizing learning rules $\left(f_{q}(q, p)<0\right)$ will lead to convergence. Indeed, for such rules $a_{11}+a_{22}<0$ and $\left(a_{11}-a_{22}\right)^{2}+4 a_{12} a_{21}<0$. Hence, both eigenvalues have negative real parts ${ }^{23}$ - our steady state is stable in continuous time. For the rules that are destabilizing $\left(f_{q}(q, p)>0\right)$, we shall have no convergence. Indeed, in this case one eigenvalue is positive, the other is negative - the linearized system is a saddle. Note that our results hold for any $\alpha \in(0,1)$.

\section{A3. Convex auditing function}

With a convex auditing function $C($.$) the myopic best response of the monitoring$ authority is no longer jumping, but is a smooth function of $q$ :

$$
\begin{aligned}
B R(q) & =C^{\prime-1}(q F) . \\
q F & =C^{\prime}(p) \\
F d q & =C^{\prime \prime}(p) d p
\end{aligned}
$$

\footnotetext{
${ }^{23}$ We do not make any statements about the corner solutions at this point.
} 
and all our insights are preserved, if the slope of the steady auditing line $d q / d p^{B R}=$ $C^{\prime \prime}() /$.$F does not exceed (in absolute terms) the slope of steady compliance line, i.e.$ if the auditing is not too convex.

Note that with convex auditing function the case of no inertia $(\alpha=1)$ in tax authority decision also generates the dynamics qualitatively similar to the one analyzed

in this paper. In particular, the stability matrix becomes $\left(\begin{array}{cc}f_{q}(q, p) & f_{p}(q, p) \\ B R^{\prime}(q) & -1\end{array}\right)$, so as long as $B R(q)$ is steep enough (again, the auditing function is not too convex), the results of proposition 3 are preserved.

\section{A4. Low information rule is stabilizing}

We use the fact that cumulative binomial distribution function can be expressed as

$$
\operatorname{Pr}\left(k \leq k^{*}\right)=\left(m-k^{*}\right)\left(\begin{array}{c}
m \\
k^{*}
\end{array}\right) \int_{0}^{1-p q} t^{m-k^{*}-1}(1-t)^{k^{*}} d t
$$

so that

$$
\frac{\partial}{\partial q} \operatorname{Pr}\left(k \leq k^{*}\right)=-p\left(m-k^{*}\right)\left(\begin{array}{l}
m \\
k^{*}
\end{array}\right)(1-p q)^{m-k^{*}-1}(p q)^{k^{*}}
$$

Then,

$$
f(q, p)=(1-q p) \operatorname{Pr}\left(k \leq k^{*}\right)-q,
$$

and

$$
f_{q}(q, p)=-p \operatorname{Pr}\left(k \leq k^{*}\right)+(1-q p) \frac{\partial}{\partial q} \operatorname{Pr}\left(k \leq k^{*}\right)-1,
$$

where all the three terms are negative for any admissible values of variables. Thus, we have shown that $f_{q}(q, p)<0$ everywhere in the domain, or, equivalently, that the rule is stabilizing.

\section{A5. Comparative statics for the low information rule No commitment (Proposition 4)}

For the steady state, using the fact that $q^{s s}=\mu$,

$$
\mu=\mu\left(1-p^{s s}\right)(1-\beta)+\beta\left(1-\mu p^{s s}\right)^{m+1},
$$

so that

$$
B d \beta+P d p^{s s}+M d m=0,
$$

where

$$
\begin{aligned}
B & :=\left(1-\mu p^{s s}\right)^{m+1}-\mu\left(1-p^{s s}\right), \\
M \quad: & =\beta\left(1-\mu p^{s s}\right)^{m+1} \ln \left(1-\mu p^{s s}\right)<0, \\
P \quad: & =-\mu\left((m+1) \beta\left(1-\mu p^{s s}\right)^{m}+1-\beta\right)<0 .
\end{aligned}
$$


We can also see that $B<0$ for large $m$, which are the interest of this model. We can conclude that

$$
\begin{aligned}
& \frac{d p^{s s}}{d m}=-\frac{M}{P}<0 \\
& \frac{d p^{s s}}{d \beta}=-\frac{B}{P}<0 .
\end{aligned}
$$

\section{Commitment (Proposition 4a)}

Totally differentiating the FOC for interior optimum (3) with respect to $m$, we get

$$
d \cdot d p^{*}=\left(\left(V-F p^{*}\right) \bar{q}_{m}^{\prime}\left(p^{*}, m\right)-F \bar{q}_{m}\left(p^{*}, m\right)\right) d m
$$

where $d<0$ is the second derivative of objective function with respect to $p$, defined in the text. Further, implicitly differentiating the steady state relation between compliance and auditing

$$
q=q(1-p)(1-\beta)+\beta(1-q p)^{m+1},
$$

we get (we further drop stars for ease of notation)

$$
\frac{d q}{d m}=\bar{q}_{m}\left(p^{*}, m\right)=\frac{\beta(1-q p)^{m+1} \ln (1-q p)}{p(m+1) \beta(1-q p)^{m}+p+\beta-p \beta}<0 .
$$

Similarly, we can implicitly differentiate the steady state relation with respect to auditing probability to get

$$
\frac{d q}{d p}=q^{\prime}=\frac{(m+1) \beta(1-q p)^{m}+1-\beta}{p \beta-p-\beta-p(m+1) \beta(1-q p)^{m}} q<0 .
$$

Finally, we can differentiate this derivative with respect to $m$ taking into account that $q$ is a function of $m$ as well:

$$
\begin{gathered}
q_{m}^{\prime}=-\frac{\beta^{2} q(1-q p)^{m}}{(p \beta-p-\beta-p x)^{2}}(1+(m+1) \ln (1-q p)) \\
+\frac{x+1-\beta}{p \beta-p-\beta-p x} q_{m}
\end{gathered}
$$

where

$$
x:=(m+1) \beta(1-q p)^{m} .
$$

Simplifying this further, we can show that the condition $q_{m}^{\prime}>0$ is equivalent to the condition

$$
-((x+1-\beta)(1-q p) x+m+1) \ln (1-q p)-\beta x>0
$$

which implicitly defines the maximal level of $p^{*}$ that would satisfy the proposition $4 \mathrm{a}$. With $\bar{q}_{m}^{\prime}>0$ and $\bar{q}_{m}<0$, the condition $V>F p^{*}$ is then sufficient for $\frac{d p^{*}}{d m}<0$. 


\section{A6. Rationalization}

From (14) we can see that for $k=0 \mathbb{E} v$ takes value

$$
v_{\min }:=\frac{v_{H} \rho\left(1-v_{H}\right)^{m}+v_{L}(1-\rho)\left(1-v_{L}\right)^{m}}{\rho\left(1-v_{H}\right)^{m}+(1-\rho)\left(1-v_{L}\right)^{m}}
$$

and for $k=m$ it takes value

$$
v_{\max }:=\frac{v_{H} \rho\left(v_{H}\right)^{m}+v_{L}(1-\rho)\left(v_{L}\right)^{m}}{\rho\left(v_{H}\right)^{m}+(1-\rho)\left(v_{L}\right)^{m}} .
$$

In particular, if $v_{H}=1$ and $v_{L}=0$, we have $v_{\min }=0$ and $v_{\max }=1$.

To see that $\mathbb{E} v$ is increasing in $k$, note that a sufficient condition for that is that

$$
\frac{(1-\rho) b\left(k, m, v_{L}\right)}{\rho b\left(k, m, v_{H}\right)}
$$

is decreasing in $k$ (in this case posterior probability assign to $v_{H}$ is increasing in $\mathrm{k}$ and posterior probability assigned to $v_{L}$ is decreasing in $k$ ). A necessary and sufficient condition for this is that

$$
\left(\frac{v_{H}}{v_{L}}\right)^{k}\left(\frac{1-v_{H}}{1-v_{L}}\right)^{m-k}
$$

is increasing in $k$. And indeed it does so for $v_{H}>v_{L}$.

\section{A7. Contagion}

From (15) we have

$$
f(q, p)=\left\{\begin{array}{cc}
\sum_{k=0}^{n-1}\left(\begin{array}{l}
n \\
k
\end{array}\right)(1-q)^{k} q^{n-k} \frac{\lambda k+n-k}{n}-q, & \text { if } p<\bar{p} \\
1-\sum_{k=1}^{n}\left(\begin{array}{l}
n \\
k
\end{array}\right)(1-q)^{k} q^{n-k} \frac{\lambda(n-k)+k}{n}-q, & \text { if } p>\bar{p} .
\end{array}\right.
$$

We know that for Bernstein polynomials

$$
\frac{d}{d t} B_{k, n}(t)=n\left(B_{k-1, n-1}(t)-B_{k, n-1}(t)\right)
$$

Redenoting $t=1-q$, we have then

$$
f(q, p)=\left\{\begin{array}{cc}
\sum_{k=1}^{n-1} B_{k, n}(t) \frac{\lambda k+n-k}{n}+B_{0, n}(t)-1+t, & \text { if } p<\bar{p} \\
t-\sum_{k=1}^{n-1} B_{k, n}(t) \frac{\lambda(n-k)+k}{n}-B_{n, n}(t), & \text { if } p>\bar{p} .
\end{array}\right.
$$

As $d q=-d t$, we have

$f_{q}(q, p)=\left\{\begin{array}{cl}\sum_{k=1}^{n-1}\left(B_{k, n-1}(t)-B_{k-1, n-1}(t)\right)(\lambda k+n-k)-1+n q^{n-1}, & \text { if } p<\bar{p} ; \\ \sum_{k=1}^{n-1}\left(B_{k-1, n-1}(t)-B_{k, n-1}(t)\right)(\lambda(n-k)+k)+n(1-q)^{n-1}-1, & \text { if } p>\bar{p} .\end{array}\right.$ 
Using the facts that

$$
\sum_{k=0}^{n} B_{k, n}(t)=1, \sum_{k=0}^{n} k B_{k, n}(t)=n t
$$

we get

$$
\begin{aligned}
n \sum_{k=1}^{n-1} B_{k, n-1}(t) & =n\left(1-(1-t)^{n-1}\right), \\
(\lambda-1) \sum_{k=1}^{n-1} k B_{k, n-1}(t) & =(\lambda-1)(n-1) t ;
\end{aligned}
$$

and (denoting $l=k-1$ ),

$$
\begin{aligned}
\sum_{k=1}^{n-1} n B_{k-1, n-1}(t) & =n\left(1-t^{n-1}\right), \\
(\lambda-1) \sum_{k=1}^{n-1} k B_{k-1, n-1}(t) & =(\lambda-1)\left((n-1) t+1-n t^{n-1}\right) .
\end{aligned}
$$

Plugging this into the expression for the derivative, we get

$$
f_{q}(q, p)=\left\{\begin{array}{cc}
n\left(1-(1-t)^{n-1}\right)+(\lambda-1)(n-1) t-n\left(1-t^{n-1}\right) & \text { if } p<\bar{p} ; \\
-(\lambda-1)\left((n-1) t+1-n t^{n-1}\right)-1+n q^{n-1}, & \\
\lambda n\left(1-t^{n-1}\right)+(1-\lambda)\left((n-1) t+1-n t^{n-1}\right) & \text { if } p>\bar{p} .
\end{array}\right.
$$

After collecting the terms, we arrive at (16).

\section{A8. Social learning}

Clearly, $l_{1}$ is the largest integer $l$ that satisfies inequality

$$
V_{c}<\left(1-\frac{1}{2}\left(1+\frac{l}{n}\right) v_{H}-\frac{1}{2}\left(1-\frac{l}{n}\right) v_{L}\right) V_{n}
$$

or explicitly

$$
l<2 n \frac{1-\frac{V_{c}}{V_{n}}-v_{L}}{v_{H}-v_{L}}-n .
$$

$l_{2}$ is the smallest integer $l$ that satisfies inequality

$$
V_{c}>\left(1-\frac{l}{2 n} v_{H}-\left(1-\frac{l}{2 n}\right) v_{L}\right) V_{n},
$$

or explicitly

$$
l>2 n \frac{1-\frac{V_{c}}{V_{n}}-v_{L}}{v_{H}-v_{L}} .
$$


The population dynamics can be then characterized by

$$
\begin{aligned}
q_{t+1}= & (1-p)^{n}+\sum_{k=0}^{n}\left(\begin{array}{l}
n \\
k
\end{array}\right)(1-q)^{k} q^{n-k} \sum_{l=1}^{l_{1}}\left(\begin{array}{l}
n \\
l
\end{array}\right)(1-p)^{n-l} p^{l} \\
& +\sum_{k=0}^{n}\left(\begin{array}{l}
n \\
k
\end{array}\right)(1-q)^{k} q^{n-k} \sum_{l=l_{1}+1}^{l_{2}-1}\left(\begin{array}{l}
n \\
l
\end{array}\right)(1-p)^{n-l} p^{l} \frac{n-k}{n} .
\end{aligned}
$$

Using the properties of Bernstein polynomials, this can be simplified to

$$
q_{t+1}=(1-p)^{n}+\sum_{l=1}^{l_{1}}\left(\begin{array}{l}
n \\
l
\end{array}\right)(1-p)^{n-l} p^{l}+q \sum_{l=l_{1}+1}^{l_{2}-1}\left(\begin{array}{l}
n \\
l
\end{array}\right)(1-p)^{n-l} p^{l} .
$$

Subtracting $q$ and taking the derivative brings about (17).

In order to satisfy assumption 1, a stabilizing behavioral rule must also possess the property $f_{p}(q, p)<0$. Rewriting $f(q, p)$ in terms of Bernstein polynomials, we have

$$
f(q, p)=(1-p)^{n}+\sum_{l=1}^{l_{1}} B_{l, n}(p)+q \sum_{l=l_{1}+1}^{l_{2}-1} B_{l, n}(p)-q
$$

Taking the derivative wrt to $p$, we have

$$
\frac{1}{n} f_{p}(q, p)=-(1-p)^{n-1}+\sum_{l=1}^{l_{1}}\left(B_{l-1, n-1}(p)-B_{l, n-1}(p)\right)+q \sum_{l=l_{1}+1}^{l_{2}-1}\left(B_{l-1, n-1}(p)-B_{l, n-1}(p)\right) .
$$

For $l_{1}=1$ and $l_{2}=3^{24}$ we have then

$$
\begin{aligned}
\frac{1}{n} f_{p}(q, p) & =-(1-p)^{n-1}+B_{0, n-1}(p)-B_{1, n-1}(p)+q\left(B_{1, n-1}(p)-B_{2, n-1}(p)\right) \\
& =-B_{1, n-1}(p)+q\left(B_{1, n-1}(p)-B_{2, n-1}(p)\right) \leq-q B_{2, n-1}(p)<0 .
\end{aligned}
$$

so assumption 1 holds.

\section{A9. Arbitrary rule with $n$ people in a match}

Consider a general deterministic $n$ persons rule without eigen bias (the rule is invariant to the distribution of types in a match). All individuals that belong to the same match exhibit the same behavior. Assume also that we have $m$ different observable types of individuals (observable is in the sense that the compliance decision can be conditioned upon them; in the example considered we have $m=2$ ). Call the number of combinations of the types in a match $M:=\left(\left(\begin{array}{c}m \\ n\end{array}\right)\right)$. It can be shown that $2^{M}$ distinct rules of this sort can be formulated, with the dynamics represented by $q_{t+1}=\sum_{i=1}^{M} \mathbb{I}_{i} Q_{t}(i)$. Here $Q(i)$ is the probability that the combination $i$ of the types

\footnotetext{
${ }^{24} l_{1}=1, l_{2}=2$ is a degenerate case whereby dynamics depend on $p$ only.
} 
occurs, and the particular rule specifies a sequence $\left(\mathbb{I}_{i}\right)_{i=1}^{M}, \mathbb{I}_{i} \in\{0,1\}$. For a rule not to be degenerate (jumping to a corner immediately), we must have

$$
\exists i, \mathbb{I}_{i} \neq 0 ; \exists j, \mathbb{I}_{j} \neq 1
$$

Let us concentrate on the endogenous types, that is audited honest, not audited honest, audited non-compliant and non-audited non-compliant. A period-to-period dynamics resulting from any rule can be generally written as

$$
\begin{aligned}
q_{t+1} & =\sum_{j=0}^{n} \sum_{k=0}^{j} \mathbb{I}_{j, k} n_{j, k}(1-q)^{n-j} q^{j}(1-p)^{j-k} p^{k}, \\
f(q, p) & =\sum_{j=0}^{n} \sum_{k=0}^{j} \mathbb{I}_{j, k} n_{j, k}(1-q)^{n-j} q^{j}(1-p)^{j-k} p^{k}-q,
\end{aligned}
$$

where $n_{j, k}$ is a fixed coefficient characterizing number of permutations for the same combination of types.

As can be seen, the function $f(q, p)$ is a polynomial of order at most $n$ in each of the arguments. Adding exogenous types (like rich-poor, self-employed - employees) does not change this result, as they enter the function in a similar fashion with simplex coefficients.

Furthermore, if we add a possibility of no rematching for $t_{0}$ periods (that is, our interaction groups remain closed over this time, which is reasonable if we think about families or very close friends), the dynamics can still be represented as

$$
q_{t+t_{0}}=\sum_{l=0}^{t_{0}} n_{t_{0}, l}\left(\sum_{j=0}^{n} \sum_{k=0}^{j} \mathbb{I}_{j, k} n_{j, k}(1-q)^{n-j} q^{j}(1-p)^{j-k} p^{k}\right)(1-p)^{t_{0}-l} p^{l}
$$

which is also a polynomial. That is why, our results also hold in the setting with stable over time interaction groups, provided the appropriate assumptions are satisfied.

Finally, a longer memory feature is somewhat cumbersome to represent in the general form, as instead of only $m$ types in a match, we have to consider $m^{r}$ types, where $r$ is the length of memory in periods. Apart from that, period-to-period dynamics will involve values of variables from $r$ periods. That is why we only refer here to 2-period memory with our endogenous types:

$$
\begin{aligned}
q_{t+1}= & \sum_{j=0}^{n} \sum_{k=0}^{j} \sum_{h=0}^{n} \sum_{l=0}^{h} \mathbb{I}_{j, k, h, l} n_{j, k, h, l}\left(1-q_{t}\right)^{n-j} q_{t}^{j}\left(1-p_{t}\right)^{j-k} p_{t}^{k} \times \\
& \times\left(1-q_{t-1}\right)^{n-h} q_{t-1}^{h}\left(1-p_{t-1}\right)^{h-l} p_{t-1}^{l} .
\end{aligned}
$$

This is still a polynomial. 


\section{A10. Commitment with full dynamic optimization}

A variation of committed authority is the one that maximizes not the steady state payoff, but the payoff over the full dynamic path. Such a problem can be then written as

$$
\begin{aligned}
& \quad \max _{\left\{p_{t}\right\}} \sum_{t=1}^{\infty} \beta^{t}\left(\left(1-q_{t}\right) V+p_{t} q_{t} F-c p_{t}\right) \\
& \text { s.t. } \quad: \quad q_{t}=q_{t-1}+f\left(q_{t-1}, p_{t-1}\right),
\end{aligned}
$$

where $0<\beta<1$ is a discount factor. The first order condition for an interior solution can be rewritten as

$$
\beta^{t}\left(q_{t} F-c\right)=\beta^{t+1} f_{p}\left(q_{t}, p_{t}\right)\left(V-p_{t+1} F\right),
$$

which is a familiar equality of marginal gain from extra auditing today (in terms of increase in the share of punished agents net of auditing costs) and marginal loss tomorrow (in terms of the change in the share of cheaters). Note that by Lemma 1 for stabilizing rules $f_{p}<0$, which for given state $q_{t}>c / F$ (noncompliance is higher than in myopic steady state) puts an upward pressure on future monitoring $p_{t+1}\left(p_{t+1}>V / F\right.$ is needed to satisfy the FOC). For destabilizing rules $f_{p}>0$, correspondingly, high noncompliance calls for low monitoring. Note also that the second order condition requires $f_{p p}\left(V-p_{t+1} F\right)<0$, so depending on the relative magnitude of auditing, the transition function shaped by the behavioral rule must be concave or convex in auditing for the interior solution to exist.

A steady state condition in this setting would be a pair $(p, q)$ such that

$$
\bar{q}(p) F-c=\beta f_{p}(\bar{q}(p), p)(V-p F),
$$

which is analogous to (3) up to the discount factor and the term $f_{p}(p, \bar{q}(p))$ instead of $\bar{q}^{\prime}\left(p^{*}\right)$. Intuitively, the authority in the text maximizes steady state payoff only and does not care about transition. Here, though from some time on the system is in steady state (or very close to it), the authority has maximized its payoff along the whole path, so it takes into account what happened before as given. The SOC is

$$
d d:=\bar{q}^{\prime \prime}(p) F-\beta\left(f_{p p}+f_{p q} \bar{q}^{\prime}(p)\right)(V-p F)+\beta f_{p}(\bar{q}(p), p) F<0 .
$$

Looking at comparative statics, we obtain

$$
\begin{aligned}
\frac{d p}{d c} & =\frac{1}{d d}<0, \\
\frac{d p}{d F} & =\frac{-\bar{q}(p)+\beta p f_{p}(p, \bar{q}(p))}{d d},
\end{aligned}
$$

with interpretation similar to the main text, except for that now the sign of $d p / d F$ is determined by how sensitive the transition function $f$ is to a change in $p$ rather than elasticity of steady state cheating. Our result that the 'deterrence hypothesis' may or may not hold remains intact. 


\section{A11. Steady Cycles}

\section{Inexistence of steady state}

Consider the steady state identified in (9):

$$
q^{s s}=\mu, p^{s s}=\bar{q}^{-1}(\mu) .
$$

By definition, we have

$$
f\left(\mu, \bar{q}^{-1}(\mu)\right)=0 .
$$

We show that this cannot be stable in discrete time even for stabilizing rules. Consider a mutation $\varepsilon>0$ to the share of noncompliant population and set the time to zero. Then $q_{0}=\mu+\varepsilon$ and $p_{0}=\bar{q}^{-1}(\mu)$. According to the behavioral rule,

$$
q_{1}=\mu+\varepsilon+f\left(\mu+\varepsilon, \bar{q}^{-1}(\mu)\right),
$$

and, since $f_{q}<0$ for stabilizing rules, we have $q_{1}<q_{0}$ - a welcome tendency. However, look at the monitoring probability:

$$
p_{1}=\alpha+(1-\alpha) p_{0}>p_{0} .
$$

Now, iterate again

$$
q_{2}=\mu+\varepsilon+f\left(\mu+\varepsilon, p_{0}\right)+f\left(\alpha+(1-\alpha) p_{0}, \mu+\varepsilon+f\left(\mu+\varepsilon, p_{0}\right)\right) .
$$

Since $f_{q}<0$ and $f_{p}<0$ for stabilizing rules by Lemma 1 , we have $q_{2}<q_{1}$. The monitoring probability now depends on the relative value of $q_{1}$ :

$$
p_{2}=\left\{\begin{array}{cc}
\alpha+(1-\alpha)\left(\alpha+(1-\alpha) p_{0}\right)>p_{1}, & q_{1}>\mu ; \\
(1-\alpha)\left(\alpha+(1-\alpha) p_{0}\right)<p_{1}, & q_{1}<\mu .
\end{array}\right.
$$

Observe that monitoring probability is a function of $\alpha$ and $p_{0}$ and in period $t$ can be represented as

$$
\sum_{j=0}^{t-1}(1-\alpha)^{j} \alpha B R\left(q_{j}\right)+(1-\alpha)^{t} p_{0}
$$

where $B R\left(q_{j}\right)$ is a zero-one variable. Clearly, $\lim _{t \rightarrow \infty} p_{t}=p_{0}$ by coincidence only. Thus, we have shown that auditing probability never comes back to its steady state value $p^{s s}$, or, equivalently, the steady state is not stable. Further, since the steady state considered is unique by construction, a stable steady state does not exist in our discrete time model.

\section{Existence of steady cycles}

To establish existence of steady state cycles, we first need to show that there exists an integer $k>0$ such that $\left(p_{t}, q_{t}\right)=\left(p_{t+k}, q_{t+k}\right)$. Since $\lim _{t \rightarrow \infty} p_{t}=\lim _{t \rightarrow \infty} p_{t+k}$ (in a little abuse of the term "limit"), we need to have

$$
p_{t+k}=\sum_{j=0}^{k-1}(1-\alpha)^{j} \alpha B R\left(q_{j}\right)+(1-\alpha)^{k} p_{t}=p_{t} .
$$


This gives us the following expression for the auditing probability that is a member of a steady cycle:

$$
p^{s}=\frac{\sum_{j=0}^{k-1}(1-\alpha)^{j} \alpha B R\left(q_{t+j}\right)}{1-(1-\alpha)^{k}} .
$$

Clearly, for the dynamics to be a cycle and not an arbitrary path returning to the same point, we also need $\left(p_{t+i}, q_{t+i}\right)=\left(p_{t+k+i}, q_{t+k+i}\right) \forall i \in[1, k-1]$. The analogous condition is then

$$
p^{s+i}=\frac{\sum_{j=i}^{k+i-1}(1-\alpha)^{j-i} \alpha B R\left(q_{t+j+i}\right)}{1-(1-\alpha)^{k+i}} .
$$

The path of best responses is a sequence of zeros and ones that is determined by whether the current level of cheating is below or above its steady state level $\mu$. This, given a starting point, is in turn determined by the behavioral rule. Note that for interior solution $p^{s}<1$ we need

$$
0<\sum_{j=0}^{k-1}(1-\alpha)^{j} \alpha B R\left(q_{t+j}\right)<1-(1-\alpha)^{k} .
$$

For $k=1$ this is never satisfied. For $k=2$ the admissible sequence of best responses is $(0,1)$ and $(1,0)$. Correspondingly, we have two values for $p^{s}$ :

$$
\begin{aligned}
p^{s} & =\frac{\alpha}{1-(1-\alpha)^{2}}, \\
p^{s+1} & =\frac{(1-\alpha) \alpha}{1-(1-\alpha)^{2}},
\end{aligned}
$$

or visa versa which could fit the bill and work as a steady cycle for an appropriate behavioral rule. An appropriate behavioral rule in this case is the one that generates $q_{t}=q_{t+2}=q^{s}$ and $q_{t+1}=q_{t+3}=q^{s+1}$ such that $q^{s}<\mu$ and $q^{s+1}>\mu$ implying

$$
\begin{aligned}
f\left(q^{s}, p^{s}\right) & =q^{s+1}-q^{s}>0, \\
f\left(q^{s+1}, p^{s+1}\right) & =q^{s}-q^{s+1}<0 .
\end{aligned}
$$

This way, we have constructed a steady cycle made of two points for a class of behavioral rules (the class is described by $q^{s}<\mu<q^{s+1} ; f\left(q^{s}, p^{s}\right)=-f\left(q^{s+1}, p^{s+1}\right)=$ $\left.q^{s+1}-q^{s}\right)$. But this is clearly inconsistent with the phase diagram (figure 1) that we have derived under assumptions made in the text. To see this, note that because $q^{s}<\mu<q^{s+1}$, the point $\left(p^{s}, q^{s}\right)$ should lie in the south-west region, and then, according to the phase diagram, the point $\left(p^{s+1}, q^{s+1}\right)$ lies in the north-west region. But then $\left(p^{s}, q^{s}\right)$ should lie in the north-east region, which is a contradiction.

We see that a cycle consistent with the phase diagram should include at least 4 points to represent 4 different regions. Starting, for instance, from the south-west region, we have the following sequence of best responses: $(0,1,1,0)$. Hence, the steady 
cycle probabilities are

$$
\begin{aligned}
p^{s} & =\alpha(1-\alpha) \frac{2-\alpha}{1-(1-\alpha)^{4}}, \\
p^{s+1} & =\alpha(1-\alpha)^{2} \frac{2-\alpha}{1-(1-\alpha)^{4}}, \\
p^{s+2} & =\alpha \frac{(1-\alpha)^{3}+1}{1-(1-\alpha)^{4}}, \\
p^{s+3} & =\alpha \frac{2-\alpha}{1-(1-\alpha)^{4}}
\end{aligned}
$$

with $p^{s+1}<p^{s}<p^{s+2}<p^{s+3}$. To complete the characterization, we need to specify the class of behavioral rules in the following way: $q_{t}=q_{t+4}=q^{s} ; q_{t+1}=q_{t+5}=q^{s+1}$; $q_{t+2}=q_{t+6}=q^{s+2} ; q_{t+3}=q_{t+7}=q^{s+3}$; such that $q^{s}<\mu ; q^{s+1}>\mu ; q^{s+2}>\mu ;$ $q^{s+3}<\mu$; implying

$$
\begin{aligned}
f\left(q^{s}, p^{s}\right) & =q^{s+1}-q^{s}>0, \\
f\left(q^{s+1}, p^{s+1}\right) & =q^{s+2}-q^{s+1}, \\
f\left(q^{s+2}, p^{s+2}\right) & =q^{s+3}-q^{s+2}<0, \\
f\left(q^{s+3}, p^{s+3}\right) & =q^{s}-q^{s+3} .
\end{aligned}
$$

Furthermore, we need $q^{s}<\hat{q}\left(p^{s}\right) ; q^{s+1}<\hat{q}\left(p^{s+1}\right) ; q^{s+2}>\hat{q}\left(p^{s+2}\right) ; q^{s+3}>\hat{q}\left(p^{s+3}\right)$. This completes characterization of a steady cycle consistent with the phase diagram.

Intuitively, the auditing probabilities in a cycle of a given length are determined by the inertia variable $\alpha$ completely. With small $\alpha$, we will need more points to include in the cycle for a given behavioral rule, because more iterations are needed to switch the best response from 0 to 1 and back.

\section{Cycle stability}

Once we have shown that we can construct a steady cycle of $k$ points, we can ask a question whether such cycle is stable. Consider our 4-cycle and perturb the share of non-compliant agents with $\varepsilon>0$ to get $q_{0}=q^{s}+\varepsilon, p_{0}=p^{s}$. Because $\varepsilon$ is small, it does not change the best response of the authority, and thus $p_{1}=p^{s+1}, q_{1}=q^{s}+\varepsilon+$ $f\left(q^{s}+\varepsilon, p^{s}\right)<q^{s+1}+\varepsilon$ for stabilizing rules. Again, because $\varepsilon$ is small and $f_{q}<\infty$, so $q_{1}-q^{s+1}=q^{s}+\varepsilon+f\left(q^{s}+\varepsilon, p^{s}\right)-q^{s}-f\left(q^{s}, p^{s}\right) \approx \varepsilon\left(1+f_{q}\right)$ is also small, so we have $p_{2}=p^{s+2}, q_{2}=q^{s}+\varepsilon+f\left(q^{s}+\varepsilon, p^{s}\right)+f\left(q^{s}+\varepsilon+f\left(q^{s}+\varepsilon, p^{s}\right), p^{s+1}\right)<q^{s+2}+\varepsilon$. Вy the same reasoning as before we can show that $q_{3}<q^{s+3}+\varepsilon$ and $q_{4}<q^{s}+\varepsilon$ and so on. Then, we are ready to conclude that the cycle is stable, if we also show that $q_{i}>$ $q^{s+i}-\varepsilon$. Because $q_{i}=q^{s+i-1}+\varepsilon+f\left(q^{s+i-1}+\varepsilon, p^{s+i-1}\right)$ if we introduce the perturbation at the $i$ th point in the cycle, we need to have $q^{s+i-1}+\varepsilon+f\left(q^{s+i-1}+\varepsilon, p^{s}\right)>q^{s+i}-\varepsilon$ or, letting $\varepsilon \rightarrow 0, f_{q}>-2$. If this condition is satisfied and the rule is stabilizing, we have a stable cycle. 